Article

\title{
Numerical Investigation of the Aerodynamic Characteristics and Attitude Stability of a Bio-Inspired Corrugated Airfoil for MAV or UAV Applications
}

\author{
Hui Tang ${ }^{1,2,3} \mathbb{C}$, Yulong Lei ${ }^{1,2, *}$, Xingzhong $\mathrm{Li}^{1,2}$ and Yao $\mathrm{Fu}^{1,2}$ \\ 1 College of Automotive Engineering, Jilin University, Renmin Street No. 5988, Changchun 130012, China; \\ tanghui15@mails.jlu.edu.cn (H.T.); whdxjx123@126.com (X.L.); fuy.jlu@163.com (Y.F.) \\ 2 State Key Laboratory of Automotive Simulation and Control, Jilin University, Renmin Street No. 5988, \\ Changchun 130012, China \\ 3 Department of Mechanical Engineering, Osaka University, 2-1 Yamada-oka, Suita-city, Osaka 565-0871, Japan \\ * Correspondence: leiyl@jlu.edu.cn
}

Received: 27 September 2019; Accepted: 20 October 2019; Published: 22 October 2019

\begin{abstract}
In this study, two-dimensional (2D) and three-dimensional (3D) numerical calculations were conducted to investigate the aerodynamic characteristics, especially the unsteady aerodynamic characteristics and attitude stability of a bio-inspired corrugated airfoil compared with a smooth-surfaced airfoil (NACA2408 airfoil) at the chord Reynolds number of 4000 to explore the potential applications of non-traditional, corrugated dragonfly airfoils for micro air vehicles (MAVs) or micro-sized unmanned aerial vehicles (UAVs) designs. Two problem settings were applied to our numerical calculations. First, the airfoil was fixed at a constant angle of attack to analyze the aerodynamic characteristics and the hydrodynamic moment. Second, the angle of attack of airfoils was passively changed by the fluid force to analyze the attitude stability. The current numerical solver for the flow field around an unsteady rotating airfoil was validated against the published numerical data. It was confirmed that the corrugated airfoil performs (in terms of the lift-to-drag ratio) much better than the profiled NACA2408 airfoil at low Reynolds number $R e=4000$ in low angle of attack range of $0^{\circ}-6^{\circ}$, and performs as well at the angle of attack of $6^{\circ}$ or more. At these low angles of attack, the corrugated airfoil experiences an increase in the pressure drag and decrease in shear drag due to recirculation zones inside the cavities formed by the pleats. Furthermore, the increase in the lift for the corrugated airfoil is due to the negative pressure produced at the valleys. It was found that the lift and drag in the 2D numerical calculation are strong fluctuating at a high angle of attacks. However, in 3D simulation, especially for a 3D corrugated airfoil with unevenness in the spanwise direction, smaller fluctuations and the smaller average value in the lift and drag were obtained than the results in 2D calculations. It was found that a 3D wing with irregularities in the spanwise direction could promote three-dimensional flow and can suppress lift fluctuations even at high angles of attack. For the attitude stability, the corrugated airfoil is statically more unstable near the angle of attack of $0^{\circ}$, has a narrower static stable range of the angle of attack, and has a larger amplitude of fluctuations of the angle of attack compared with the profiled NACA2408 airfoil. Based on the Routh-Hurwitz stability criterion, it was confirmed that the control systems of the angle of attack passively changed by the fluid force for both two airfoils are unstable systems.
\end{abstract}

Keywords: 2D and 3D corrugated airfoil; low Reynolds number; aerodynamic characteristics; attitude stability; MAVs or UAVs 


\section{Introduction}

For the flow around the wings or airfoils, experiments using wind tunnels and numerical simulations have been extensively conducted. In particular, with the increase in speed and size of aircraft, there are many studies in the high Reynolds number range with Reynolds number of $10^{6}$ or more, where Reynolds number (Re) is based on flight velocity and airfoil chord length. On the other hand, in recent years, micro-sized unmanned aerial vehicles (UAVs) or micro air vehicles (MAVs) have been widely used in various places such as performing tasks in confined spaces or in hazardous environments, being a sensor for disaster investigation and photography, and payload delivery services [1-3]. A most recent investigation showed that the greenhouse emissions resulted from the freight industry might be significantly reduced by UAVs and MAVs delivery [4]. However, the flow field and aerodynamic characteristics around the airfoils of MAVs or UAVs are significantly different from those of large passenger aircraft because of the low Reynolds number region ( $\operatorname{Re} \leq 10^{4}$ ). In the high Reynolds number range, the inertial force is dominant and the disordered vortex is generated and the flow becomes unstable. In the low Reynolds number range, the viscous force is dominant, and the flow is smooth and stable. As a result, the aerodynamic performance of a streamlined airfoil optimized for traditional macro-scale aircraft at high Reynolds number would degrade significantly when Reynolds number is reduced for the MAVs or UAVs [5-7]. Therefore, there is an important need to redesign the conventional streamlines airfoil for the application of MAVs or UAVs to achieve better aerodynamic performance in low Reynolds number range.

It has been found that the typical Reynolds number of dragonflies can range from 100 to 10000 and can be classified as low Reynolds number flow regime [8]. There are flapping and gliding flight modes for the dragonfly, and they fly in combination [9]. Flapping is used by many insects and allows them to move forward, climb and hover. However, flapping requires a lot of energy, so it cannot last for a long time, and gliding flight is an advantageous flight mode as it requires virtually no effort from the dragonfly [10]. Of all the natural fliers, dragonflies stand out for its efficient gliding flight, that is, they are capable of gliding for 40 chords and up to $30 \mathrm{~s}$ without any appreciable loss in altitude with a single flapping [11]. Unlike other bio-inspired airfoil cross-sections, which are simple and smooth cambered surfaces, dragonfly wing's cross-section is found to be a corrugated surface. Many investigations have been performed to study this kind of wings. According to traditional airfoil design principles for high Reynolds number, the corrugated wing will have very poor aerodynamic performance, that is, high drag and low lift. However, several investigations showed that corrugated dragonfly wings would perform as well as and sometimes better than smooth technical airfoils in the low Reynolds number regime in which a dragonfly often flies [9,12-18]

Several hypotheses have been developed to show the underlying mechanism of the unexpected improvement in the aerodynamic performance of the corrugated dragonfly airfoils/wings over conventional smooth airfoils. Rees [10] and Rudolph [12] showed that the corrugated airfoil can function as a streamlined airfoil since the fluid flow will be trapped between the corrugation valleys through the formation of stagnancy or rotation when it is passing the corrugated airfoil. Newman et al. found that the improved performance can be associated with the earlier reattachment of the flow separation on the corrugated airfoil. As the angle of attack increase, the separated flow from the leading edge forms a separation bubble. The separated flow reattaches sooner due to the corrugations compared with the smooth airfoils. Meanwhile, Rudolph [12] suggested that corrugations can delay the flow separation near the leading edge of the corrugated airfoil at higher angles of attack. Kesel et al. [9] found that the negative pressure that produced at the valleys of the corrugated airfoil is the key cause of the improvement of lift. Despite different explanations about the underlying mechanism for the improved aerodynamic performance, the studies unanimously agree that the corrugated dragonfly wings work well in low Reynolds number regimes, which naturally points to the potential applications of employing such bio-inspired airfoils/wings in UAVs or MAVs.

On the other hand, the size of MAVs or UAVs is increasingly smaller and lends them to low altitude, close-in support operations. Low altitude flights pose a challenging operational environment 
for MAVs/UAVs [19-21]. One particular challenge is ensuring sufficient attitude ability in the presence of significant turbulence $[19,22]$. It should be noted that there are many experimental and numerical investigations on the aerodynamic performance of the corrugated dragonfly airfoils/wings, while few studies have so far been conducted to investigate the unsteady aerodynamic characteristics and the attitude stability of the corrugated airfoils. Meanwhile, the corrugated wings can be treated as elastic bodies rather than only rigid bodies. In fact, the natural corrugated wings of dragonflies are produced by an elastic film and rigid rod. When gilding, the wings will twist around the spanwise direction axis due to the fluid force on the wing surface. Therefore, the attitude of the corrugated airfoil is also changed for the elastic wings due to the hydrodynamic moment on the corrugated airfoil. Then, the lift and drag of the elastic wings are changed compared with that of the rigid wings due to the deformation wings (in terms of changing the attitude of the corrugated airfoils). However, studies on the hydrodynamic moment and attitude stability of the corrugated airfoils are even fewer. From the above considerations and reasons, it is therefore necessary and important to investigate the unsteady aerodynamic characteristics, hydrodynamic moment and attitude stability of the corrugated dragonfly airfoil for MAVs or UAVs applications.

In this study, two-dimensional and three-dimensional numerical simulations were conducted to investigate the aerodynamic characteristics, especially the unsteady aerodynamic characteristics and attitude stability, of a bio-inspired corrugated airfoil compared with a smooth-surfaced airfoil (NACA2408 airfoil) at the chord Reynolds number of 4000 to explore the potential applications of non-traditional, corrugated dragonfly airfoils for small MAVs or UAVs designs. The current numerical solver for the flow field around an unsteady rotating airfoil when the angle of attack is passively changed by the fluid force was validated in comparison with the published numerical data. The aerodynamic characteristics of the corrugated airfoil and smooth-surfaced airfoil were compared. In particular, the unsteady behaviors of lift coefficient and drag coefficient for both two kinds of airfoils depending on the angle of attack were considered. The effect of three-dimensional simulation on suppressing the fluctuation of the lift coefficient and drag coefficient was investigated. To evaluate the attitude stability, the hydrodynamic moments of the airfoil and the vibration of the angle of attack as the angle of attack was passively changed by fluid force were studied. The destabilization of the attack angle of the corrugated airfoil and smooth-surfaced airfoil was also studied by modeling the motion of a rotationally supported airfoil that receives fluid force and using the Routh-Hurwitz stability criterion.

\section{Numerical Method}

In this study, two problem settings were applied. In the first, the airfoil was fixed at a constant angle of attack to analyze the unsteady aerodynamics of the corrugated airfoil and smooth-surfaced airfoil. In the second, to consider the influence of the fluid force acting on the airfoil surface, the angle of attack of airfoils was passively changed by the fluid force to analyze the attitude stability. When the case of the angle of attack was passively changed by the fluid force, a moving grid technical adapted to the surface of airfoils was used.

The basic equations are the continuity equation and the Arbitrary Lagrangian-Eulerian (ALE) form Navier-Stokes equation for an incompressible Newtonian fluid. The density and viscosity of flow are assumed to be constant. All variables are non-dimensionalized by chord length $L$ and the mainstream velocity $U_{\text {uni }}$. General curvilinear coordinates $(\xi, \eta, \varsigma)$ were used for all computations due to applying the boundary-fitted-grid. Thus, the governing equations can be written as:

$$
\begin{gathered}
\frac{1}{J} \frac{\partial}{\partial \xi^{k}}\left(J U^{k}\right)=0 \\
\frac{\partial\left(u_{i}\right)}{\partial t}+\frac{1}{J} \frac{\partial}{\partial \xi^{k}}\left[u_{i}\left(J U^{k}-J V^{k}\right)\right]=-\frac{1}{J} \frac{\partial}{\partial \xi^{k}}\left(J \frac{\partial \xi^{k}}{\partial x_{i}} p\right)+\frac{1}{R e J} \frac{\partial}{\partial \xi^{k}}\left(J \frac{\partial \xi^{k}}{\partial x_{m}} \frac{\partial \xi^{l}}{\partial x_{m}} \frac{\partial u_{i}}{\partial \xi^{l}}\right),
\end{gathered}
$$


where $x_{i}$ represents the Cartesian coordinate component, $\xi^{k}$ is the general coordinate component, $J$ is the Jacobian of the coordinate transformation, $u_{i}$ is the velocity component in the general coordinate, $U^{k}$ is the contravariant velocity component, $U^{k}$ is the moving speed component of the grid point in the general coordinate, $p$ is the pressure, and $R e$ is the Reynolds number (as in $\rho U_{u n i} L / v$, where $v$ is molecular viscosity). For the case of the angle of attack is passively changed by the fluid force, the angle-of-attack variation due to the fluid force is expressed by the following equation of motion in the pitching direction:

$$
-\frac{d^{2} \alpha}{d t^{2}}=\frac{1}{2} u_{u n i}^{2} S\left(C_{M}-C_{M 0}\right)
$$

where $I, C_{M}$, and $C_{M 0}$ are the inertia moment and the moment coefficient of the force in the pitching direction applied to the airfoil at the midpoint of the upper and lower airfoil surfaces with a chord length of $25 \%$ from the airfoil leading edge, $S$ is the effective area, and $\alpha$ is the angle of attack, which is opposite to the direction defined by the moment $M$ of the fluid force. Thus, a negative sign is used on the left-hand side of Equation (3).

The non-dimensionalized governing equations are discretized employing a cell-centered, collocated arrangement where all physical variables are located at the cell center and the contravariant components are located at the cell-face center. A fourth-order central finite-difference discretization scheme was used for discretizing the space. The fractional method was selected for coupling the continuity equation and the pressure field [23]. In the numerical calculation of the Navier-Stakes equation, the second-order Adams-Bashforth method was used for the time evolution of the convective terms, while the viscous terms were discretized with the second-order Crank-Nicolson method. which eliminates the viscous stability constraint. The backward Euler method was used on the pressure term. A Neumann boundary condition was conducted on the pressure correction step at all boundaries. The Poisson equation, being the most time-consuming part for calculation of the incompressible flow, was solved with the residual cutting method [24], which employs a Gauss-Seidel line-SOR (successive over-relaxation) smoother. The present numerical method and computer program have been tested extensively in several turbulent flows [25-27]. For the case when the angle of attack was passively changed by the fluid force, the Runge-Kutta method with second-order accuracy was employed for the time evolution of Equation (3).

\section{Computational Setup}

In the numerical calculation, a corrugated airfoil that models the front cross-section of an actual dragonfly was used, and its shape is shown in Figure 1. The bent shape was measured by other researchers $[9,28]$ as a cross-section of the center part of the forehead of the dragonfly wing. The ratio of $\tau / L$ was about $7.5 \%$ and the thickness of the corrugated airfoil was about $1 \%$ of the chord length. For the sake of convenience of calculation, the leading and trailing edges were sharp. In addition, for comparison with the corrugated airfoil, NACA2408 airfoil (see Figure 2) was selected from the results of mean streamlines around the corrugated airfoil, in which fluid flowing over the corrugated airfoil would be trapped between the corrugation valleys, resulting in the corrugated airfoil functioning as a streamlined airfoil. That is, NACA2408 airfoil matches the functioned streamlined airfoil for the corrugated airfoil, where the maximum thickness of the smooth-surfaced airfoil is $8 \%$ of the chord length.

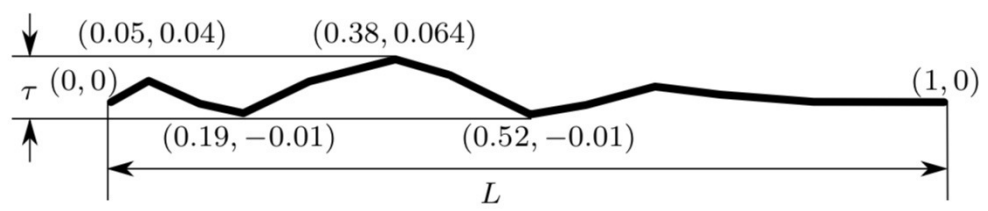

Figure 1. Profile of corrugated airfoil. 
Figure 2. Profile of NACA2408 airfoil.

The computational domain and boundary conditions for corrugated airfoil and NACA2408 airfoil are shown in Figure 3. There were two cases: two-dimensional (2D) computational domain and three-dimensional (3D) computational domain. The three-dimensional domain was used as an example to introduce the computational setup of the present study. Removing the 3D computational setup in the spanwise direction, a 2D computational setup was obtained. A Cartesian coordinate system was used to define $x$ in the mainstream direction, $z$ in spanwise direction and $y$ in the vertical direction (perpendicular to $x$ and $y$ ). Actually, all computations were conducted on a general curvilinear coordinate system $(\xi, \eta, \zeta)$, in which $\xi$ means the direction following the mainstream surface of the airfoil, $\eta$ is the direction away from the surface of airfoil, and $\zeta$ is the same as the direction of $z$. In this study, the computational grid was generated using Pointwise, a high-quality mesh generator for fluid analysis. The boundary-fitted grid of H-type was applied in the $x-y$ plane. The computational grids were regenerated as the airfoil moved. The grid points at the surrounding boundary in the calculation region in Figure 3 were fixed. The grid points on the moving airfoil surface were obtained by using Transfinite interpolation and the grid points inside the computational domain were arranged so that the grid lines were orthogonal at the boundary of the computational domain by solving an elliptic equation for general coordinate system variables [29]. Since the numerical calculation related to the problem of the fixed angle of attack did not deal with the movement of the object boundary, the moving speed of the grid $V^{k}$ (see Equation (2)) was set to zero and the computational grid was not regenerated. Figure 4 shows the calculation grid near the object boundary, an enlarged view of the leading edge of the airfoil.

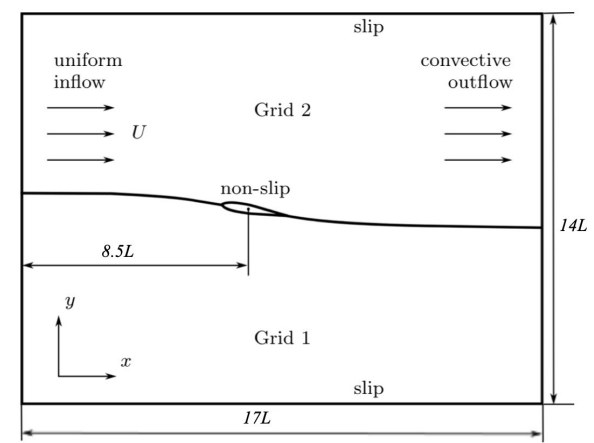

(a)



(b)

Figure 3. Computational domain and boundary conditions: (a) two dimensional; and (b) three dimensional. 


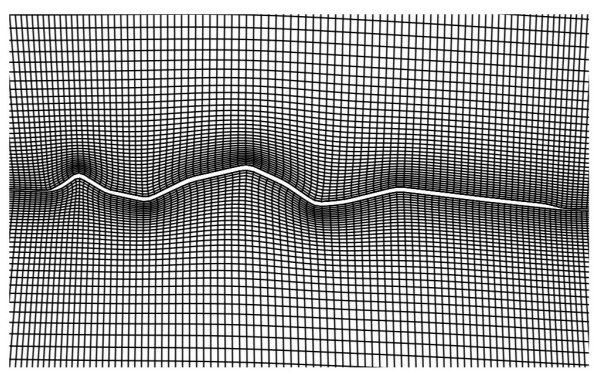

(a)

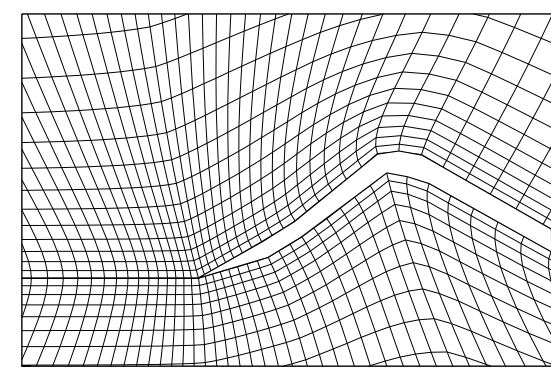

(b)

Figure 4. Computational grid: (a) corrugated airfoil; and (b) around leading edge.

The computational domain size was defined as follows: the length of the streamwise direction, wall-normal direction, and spanwise direction were $17 \mathrm{~L}, 14 \mathrm{~L}$, and $0.6 \mathrm{~L}$, respectively. The center point of the airfoil was fixed at a position $8.5 \mathrm{~L}$ from the inflow side. As shown in Figure 3, the inflow was a uniform stream without disturbance. Thus, the turbulence was developed in the boundary layer around the airfoil after the transition. The outflow boundary condition was defined as the convective boundary condition. In the spanwise direction, the periodic boundary condition was used. The slip boundary conditions were imposed on the upper and lower boundaries of the calculation region. The nonslip boundary condition was applied at the surface of the airfoil. Neumann condition with zero gradients was applied to the pressure boundary condition of inflow, outflow, top, and bottom. The computational parameters of all possible cases conducted in this study are summarized in Table 1. The Reynolds number defined by $R e=U L / v=4000$ matches the low Reynolds number range where dragonflies usually operate. Two-dimensional and three-dimensional simulations were conducted and compared to investigate the unsteady aerodynamic characteristics of the corrugated airfoil. The 2D and 3D numerical calculations at the angle of attack from $0^{\circ}$ to $12^{\circ}$ were performed to investigate the unsteady behavior of lift and drag coefficients depending on the angle of attack. The 2D simulations at the angle of attack from $-6^{\circ}$ to $6^{\circ}$ were conducted to study the attitude stability of the corrugated airfoils. In Table $1, N_{\alpha}$ and $\Delta_{\alpha}^{+}$denote the number of grid points and grid spacing in the $\alpha$ direction. The superscript + means the wall unit, that is,

$$
\Delta_{\alpha}^{+}=\frac{u_{\tau \Delta_{\alpha}}}{v}, \quad u_{\tau}=\sqrt{\frac{\tau_{w}}{\rho}},
$$

where $u_{\tau}$ is the averaged local wall friction velocity and $\tau_{w}$ denotes the wall stress.

Table 1. Computational parameters. (The superscript + means the wall unit.)

\begin{tabular}{cccccc}
\hline Case & $R \boldsymbol{e}$ & Angle of Attack & $L_{x} \times L_{y} \times L_{z}$ & $N_{x} \times N_{y} \times N_{z}$ & {$\left[\Delta_{x}^{+}, \Delta_{y}^{+}, \Delta_{z}^{+}\right]_{\text {min }}$} \\
\hline 2-D & 4000 & $-6^{\circ}-12^{\circ}$ & $17 L \times 14 L$ & $1626 \times 255$ & $9.8,0.44$ \\
3-D & 4000 & $0^{\circ}-12^{\circ}$ & $17 L \times 14 L \times 0.6 L$ & $1626 \times 255 \times 100$ & $9.8,0.44,6.2$ \\
\hline
\end{tabular}

\section{Validation Case}

To validate the current numerical solver when the angle of attack is passively changed by the fluid force, simulations of the flow field around an unsteady moving airfoil were performed and compared to the published results of Kaneko et al. [30]. The calculation target was NACA0010 airfoil that performs a heaving motion in a sufficiently wide space at an angle of the attack $0^{\circ}$, and the thrust coefficient acting on the airfoil by hydrodynamic force was compared. The airfoil analyzed in this study was a rigid body, and the forcing motion expressed by the following equation was given to the heaving airfoil:

$$
h=-h_{a m}^{*} \sin \left(2 \pi f^{*} t^{*}\right),
$$


where $*$ is a non-dimensional variable with the chord length $\mathrm{L}$ and the airfoil forward propulsion speed $U$, and $h_{a m}^{*}$ and $f^{*}$ are the amplitude and frequency of the heaving motion. The center of motion $O$ was $b=0.2 L$ measured from the blade leading edge. Figure 5 illustrates the movement variables. The computational domain and boundary condition of the validation case are shown in Figure 6, and the computational parameters are shown in Table 2. As shown in Figure 6, the grid width in wall unit was obtained on the suction side at the mid-chord position.



Figure 5. Parameters for the airfoil movements in the case of validation.

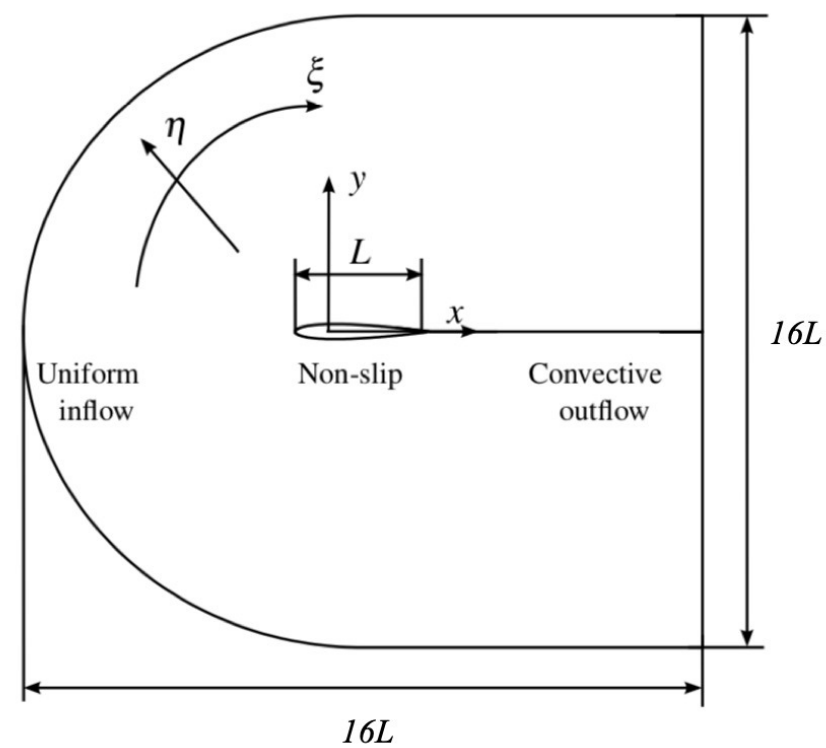

Figure 6. Computational domain and boundary condition of the validation case.

Table 2. Computational parameters of validation case. (The superscript * is a non-dimensional variable with the chord length $\mathrm{L}$ and the airfoil forward propulsion speed $U$.)

\begin{tabular}{ccccccc}
\hline $\boldsymbol{R} \boldsymbol{e}$ & Angle of Attack & $\boldsymbol{h}_{a m}^{*}$ & $f^{*}$ & $\boldsymbol{L}_{x} \times \boldsymbol{L}_{y}$ & $\boldsymbol{N}_{x} \times \boldsymbol{N}_{y}$ & $\boldsymbol{\Delta}_{x}^{+}, \boldsymbol{\Delta}_{y}^{+}$ \\
\hline 10,000 & $0^{\circ}$ & 0.05 & 0.56 & $40 L \times 30 L$ & $1800 \times 240$ & $40,1.2$ \\
\hline
\end{tabular}

For the results of the validation case, as expressed in Equation (5), the heaving motion treated in this study had periodicity, thus the lift coefficient and drag coefficient were evaluated by the phase average value. The number of samples for phase averaging was 4 . Figure 7 shows the distribution of the thrust coefficient $C_{T}$ with respect to the heaving position $h^{*}$. It can be confirmed that the calculation result by the current method is in good agreement with the numerical results by Kaneko's method [30]. Furthermore, when the airfoils were heaving and pitching, the separation vortex generated at the leading edge of the airfoil may merge with the vortex generated from the trailing edge to form a reverse Karman vortex street in the wake. It was reported by Anderson et al. [31] that the airfoil achieves high propulsion efficiency under conditions where the inverted Karman vortex is formed. 
For this reason, paying attention to the vorticity distribution around the airfoil during the upstroke (see Figure 8), no vortex separation or reverse Karman vortex street was observed at the leading edge of the blade. This observation was considered to be reasonable because drag was dominant, as shown in Figure 7.

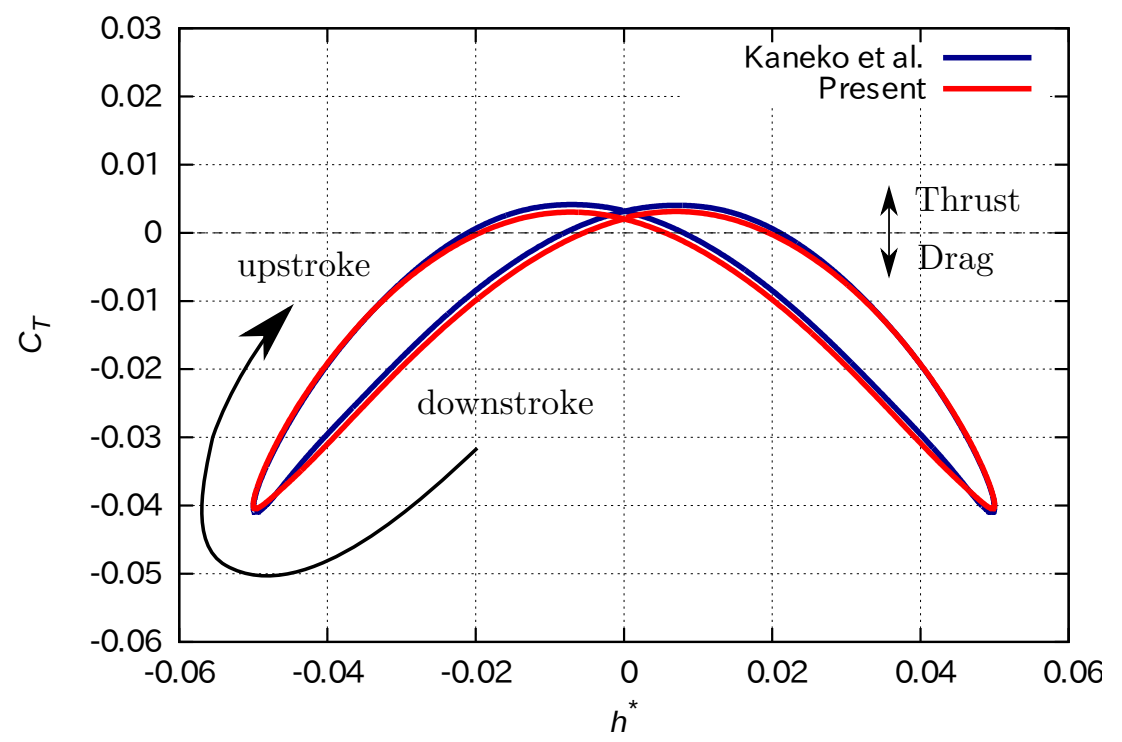

Figure 7. Periodic variation of the thrust coefficient of a heaving and pitching NACA0010 airfoil.



Figure 8. Periodic variation of the thrust coefficient of a heaving and pitching NACA0010 airfoil.

\section{Results and Discussion}

\subsection{Aerodynamic Characteristics under Two-Dimensional Calculation}

The flow field around the corrugated airfoil at chord Reynolds number $R e=4000$ with an angle of attack of $\alpha=2^{\circ}$ was taken up as a characteristic example to analyze the aerodynamics of a corrugated airfoil in relation to the flow field around it at the low Reynolds number flow and low angle of attack. Figures 9 and 10 show the time-averaged streamlines and the pressure distribution generated by the corrugated airfoil at angle of attack of $2^{\circ}$, Reynolds number $R e=4000$, respectively. It can be seen that there is a trapped vortex in each cavity of the corrugated airfoil which causes the overall flow 
to resemble that past the smooth-surfaced airfoil. This observation is in line with the experimental and numerical studies of Rees [10], Newman et al. [12] and Vargas et al. [15], who hypothesized that flow would behave in such a way due to the trapped vortex inside each cavity. Here, NACA2408 airfoil matches the functioned streamlined airfoil for the corrugated airfoil. Thus, the NACA2408 airfoil was selected to compare the smooth-surfaced airfoil with the corrugated airfoil with respect to its aerodynamic characteristics.

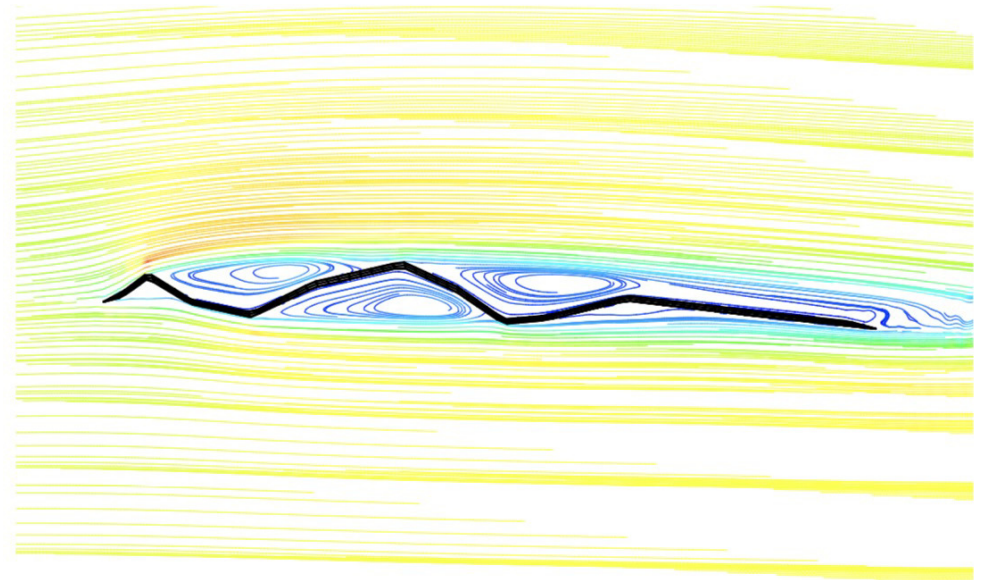

Figure 9. Mean streamlines around a corrugated airfoil at angle of attack of $2^{\circ}$ and $R e=4000$.

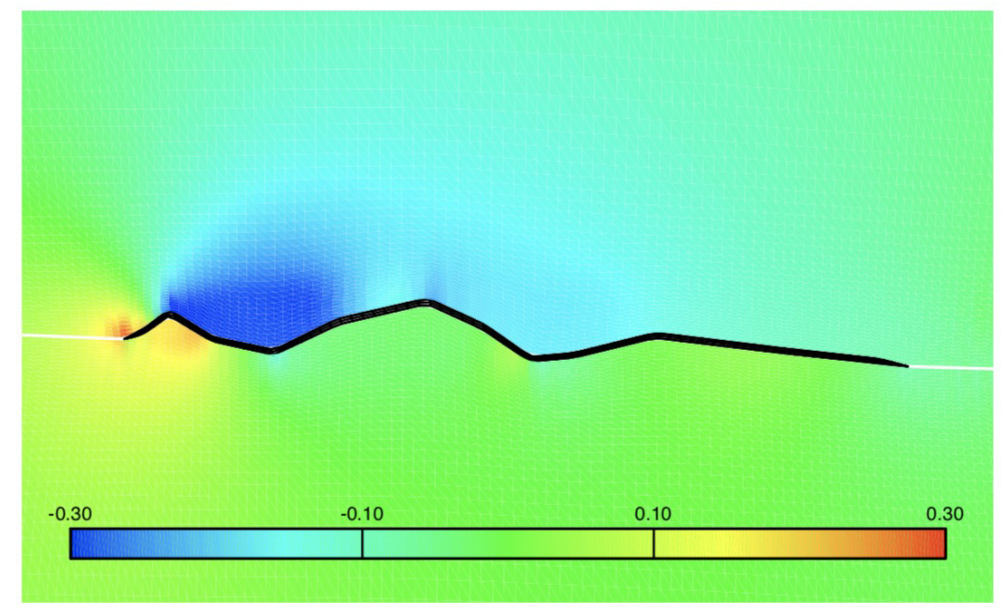

Figure 10. Mean pressure distribution of a corrugated airfoil at angle of attack of $2^{\circ}$ and $\operatorname{Re}=4000$.

The key quantities that were to be examined for the corrugated and smooth-surfaced airfoils were the coefficient of lift $\left(C_{L}\right)$ and coefficient of drag $\left(C_{D}\right)$, which can be defined as:

$$
C_{L}=\frac{F_{L}}{1 / 2 \rho U_{\infty}^{2} A}, \quad C_{D}=\frac{F_{D}}{1 / 2 \rho U_{\infty}^{2} A},
$$

where $F_{L}$ and $F_{D}$ are lift and drag force, $\rho$ is the fluid density, $U_{\infty}$ is the velocity of body relative to the fluid, and $A$ is the projected area of the airfoil. Thus, the lift-to-drag ratio is $C_{L} / C_{D}$. Table 3 shows the comparison of the lift-to-drag ratio, lift coefficient, drag coefficient, and the breakdown of lift coefficient and drag coefficient for the pressure and friction stress components with respect to the corrugated airfoil and NACA2408 airfoil at the angle of attack of $2^{\circ}, R e=4000$. Here, the superscripts $p$ and $f$ mean the contribution of pressure and friction stress, respectively. For example, $C_{L}^{p}$ denotes the pressure component of the lift coefficient. Table 3 confirms that the corrugated exhibits better aerodynamic performance than the NACA2408 airfoil at a low angle of attack, that is the lift-to-drag 
ratio of the corrugated airfoil is higher, which is in accordance with the numerical results of Luo and Sun [14] and Vargas et al. [15]. Furthermore, the corrugated airfoil could provide larger lift than the profiled NACA2408 airfoil at a low angle of attack, while the drag generated by the corrugated airfoil and the profiled NACA2408 airfoil is almost the same. Meanwhile, it can be seen that from the perspective of the pressure and friction stress, the breakdown components of the lift and drag coefficient with respect to the corrugated and profiled NACA2408 airfoils are different in Table 3. First, for the lift coefficient, there is almost no difference from the frictional contributions with respect to the corrugated airfoil and the profiled NACA2408 airfoil, while the pressure component of the lift coefficient significantly increases for the corrugated airfoil. Through analysis of the pressure distribution of the corrugated airfoil in Figure 10, the reason can be found: the negative pressure produced at the valleys of the corrugated airfoil contributes to the increased lift. Second, for the drag coefficient, the pressure contribution accounts for about $70 \%$ for the corrugated airfoil, whereas the frictional contribution accounts for about $70 \%$ for the profiled NACA2408 airfoil. The reason the pressure contribution of the drag coefficient for the corrugated airfoil is large is that the downstream side is low pressure and the upstream side is high pressure, as can be seen in Figure 10. On the other hand, the frictional contribution of drag for the profiled NACA2408 airfoil is large because of the large surface area facing the external flow.

Table 3. The lift and drag coefficients of a corrugated airfoil at angle of attack of $2^{\circ}, R e=4000$ and its breakdown.

\begin{tabular}{cccccccc}
\hline Case & $C_{L} / C_{D}$ & $C_{L}$ & $C_{D}$ & $C_{L}^{p}$ & $C_{L}^{f}$ & $C_{D}^{p}$ & $C_{D}^{f}$ \\
\hline Corrugated airfoil & 5.83 & 0.338 & 0.058 & 0.335 & 0.003 & 0.041 & 0.017 \\
NACA2408 airfoil & 3.47 & 0.205 & 0.059 & 0.203 & 0.002 & 0.018 & 0.041 \\
\hline
\end{tabular}

Figure 11 shows the values of the lift and drag coefficient of the two-dimensional corrugated airfoil and the profiled NACA airfoil at Reynolds number $R e=4000$ when the angle of attack is varied from $0^{\circ}$ to $12^{\circ}$. The lift coefficient and drag coefficient were averaged over time after the flow field was fully developed. Here, the results of NACA2406 and NACA2410 airfoils are also shown in Figure 11 to confirm if the thickness of the airfoil significantly affects the aerodynamic characteristics. The thickness of NACA2406 airfoil and NACA2410 airfoil are $6 \%$ and 10\% of the chord length, respectively. In general, it is said that the smaller is the airfoil surface area, the better is the aerodynamic performance in the flow field where the viscosity is dominant. Our result truly shows that the thinner are the airfoils, the higher is the lift and the lower is the drag. However, this kind of differences for the lift and drag between the NACA2406, NACA2410 and NACA2410 airfoils are very small. The error bars in Figure 11 indicate the maximum and minimum values of the fluctuations of the lift and drag coefficient. For the lift coefficient, the corrugated airfoil shows larger value than NACA2408 airfoil at all angle of attack, while the fluctuations for the corrugated airfoil are larger as well compared to the profiled NACA2408 airfoil when the angle of attack is increased. For the drag coefficient, the corrugated airfoil and profiled NACA2408 airfoil perform same value at low angles of attack, but the corrugated airfoil show larger value at angle of attack of $6^{\circ}$ and above. 


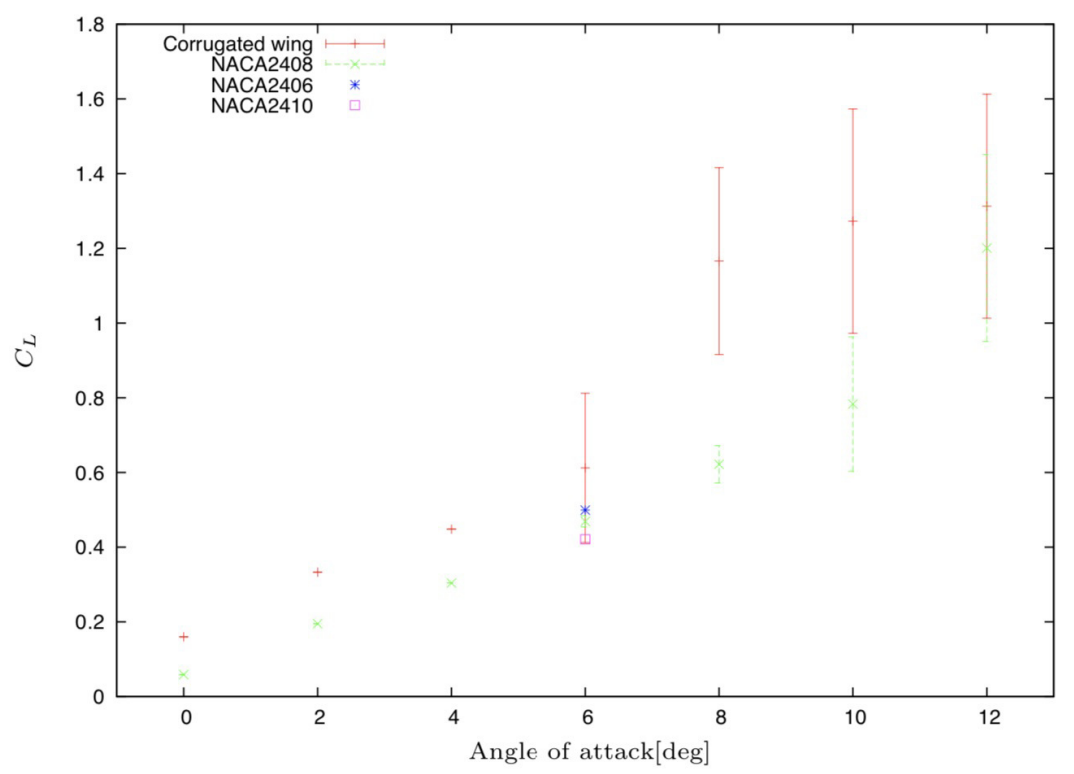

(a)

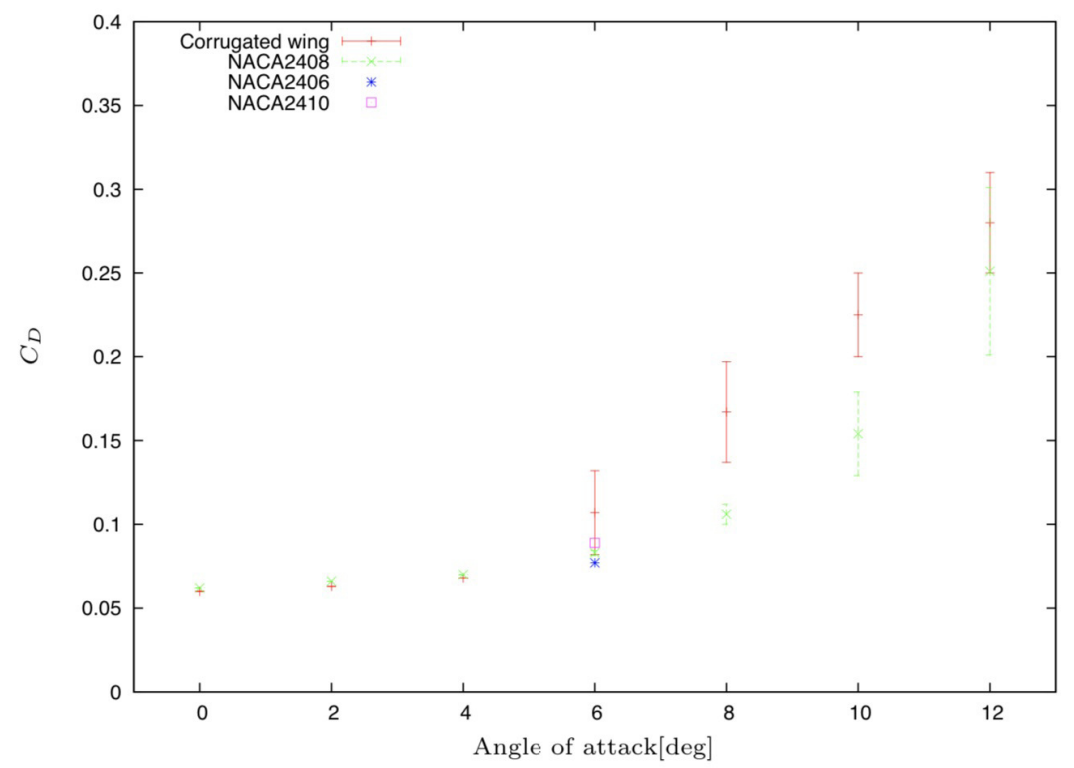

(b)

Figure 11. The mean lift and drag coefficient of the two dimensional corrugated airfoil and the profiled NACA airfoil at $R e=4000$ with varying angle of attack: (a) lift coefficient; and (b) drag coefficient.

When the angle of attack of the airfoil is large and the lift and drag are fluctuating, as Nakae et al. [32] pointed out, it is possible that the flow around the airfoil was originally three-dimensional, so the result of two-dimensional simulations of the flow around an airfoil is considered to be non-physically large and fluctuating. When the angle of attack is small, the state of the flows around an airfoil is almost steady. However, when the angle of attack increases, a separation vortex is generated on the airfoil surface, and both the lift and drag change as it flows backward. In the two-dimensional calculation, the vorticity component is fixed to only one component, so there is no room for the vortex to become three-dimensional. A strong and continuous transverse vortex is generated on the upper surface of the airfoil, which is different from the actual flow field around an airfoil, as shown in Figures 12 and 13, which show the instantaneous mainstream velocity field and vorticity around NACA2408 airfoil at angle of attack of $8^{\circ}, R e=4000$. For this reason, the two-dimensional calculation is not sufficient at high angles of attack, and it is considered that 
three-dimensional calculation is necessary at angles of attack of $6^{\circ}$ or more for corrugated airfoil and angles of attack of $8^{\circ}$ or more for NACA2408 airfoil.



Figure 12. Instantaneous velocity field around NACA2408 airfoil at angle of attack of $8^{\circ}$ and $R e=4000$.

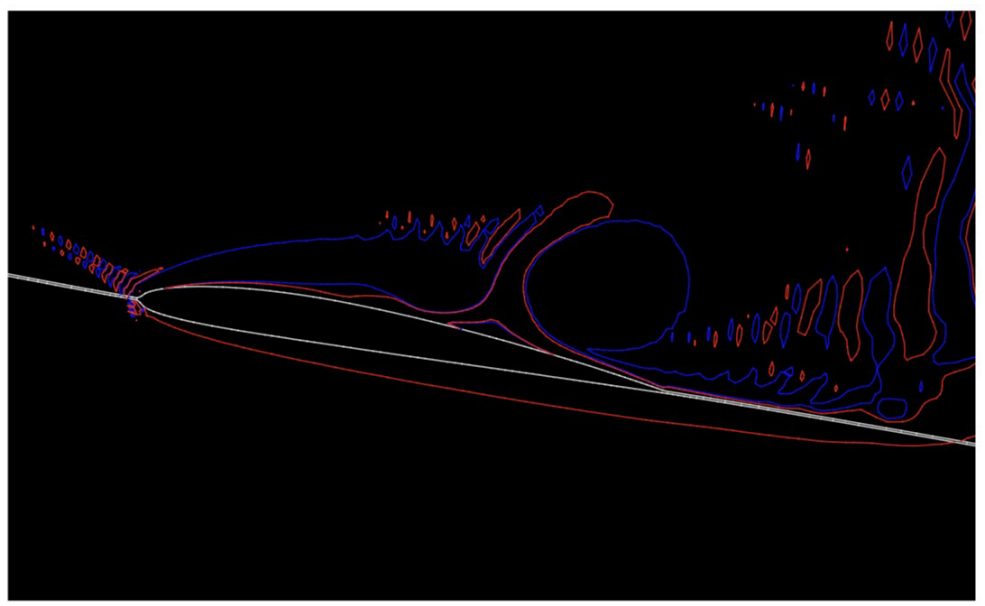

Figure 13. Instantaneous vorticity contours around NACA2408 airfoil at angle of attack of $8^{\circ}$ and $\operatorname{Re}=4000$,

\subsection{Aerodynamic Characteristics under Three-Dimensional Calculation}

Figure 14 shows the time history of lift and drag coefficient of the two and three-dimensional corrugated airfoils at $R e=4000$ with the angle of attack of $8^{\circ}$. The flows around the corrugated airfoils are sufficiently developed at a dimensionless time of about 20, and the course in Figure 14 until the dimensionless time of 80 is shown. It can be seen that the two and three-dimensional calculations for the corrugated airfoils have a distinct time history. For instance, in 2D calculations, the lift and drag coefficient are quasi-stationary states, that is the lift and drag both attain an oscillatory state, whereas, in $3 \mathrm{D}$ calculations, they are unsteady. It can also be seen that the results of the three-dimensional calculation show a smaller vibration (fluctuation) and a smaller average value in the lift and drag coefficient than the two-dimensional calculation. 


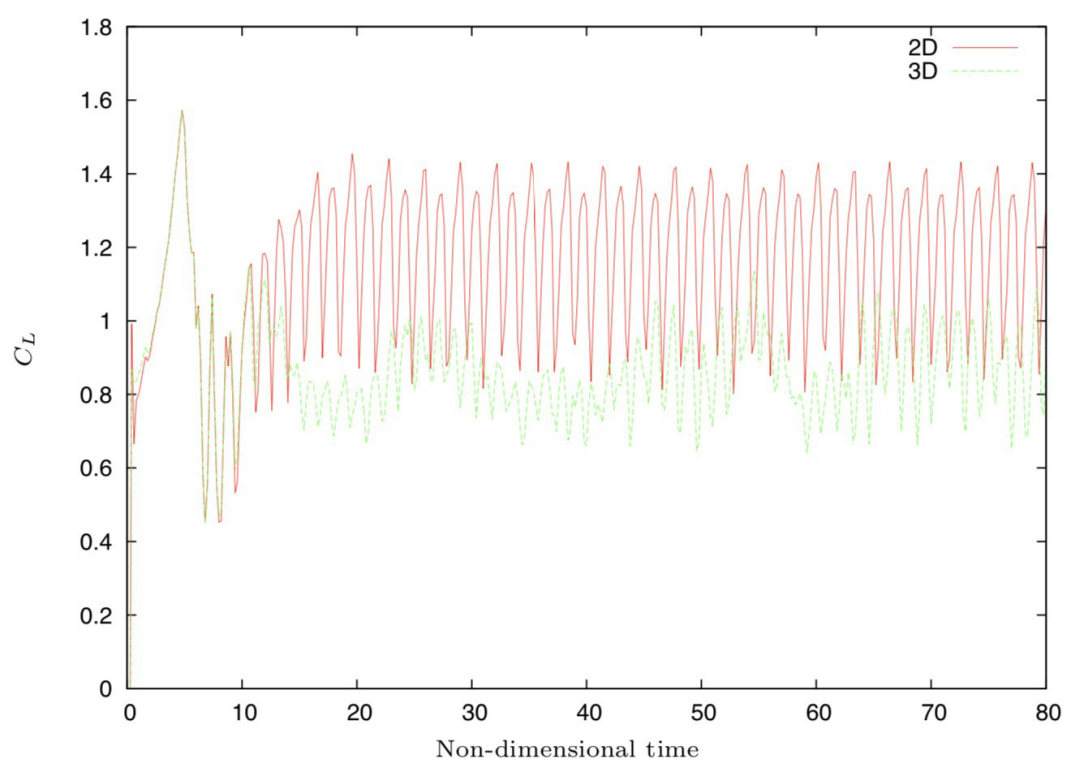

(a)



(b)

Figure 14. Time history of lift and drag coefficient of 2D and 3D calculations for the corrugated airfoil at $R e=4000$ with angle of attack of $8^{\circ}:$ (a) lift coefficient; and (b) drag coefficient.

Figure 15 shows the instantaneous velocity field on the upper surface of the corrugated airfoil at angle of attack of $8^{\circ}, R e=4000$. Figure 15 a shows the velocity field viewed from diagonally upward, while Figure 15b shows the velocity field viewed from the front side. From this result, it was confirmed that the flow field around the corrugated airfoil is three-dimensional, especially in the separation shear layer. 


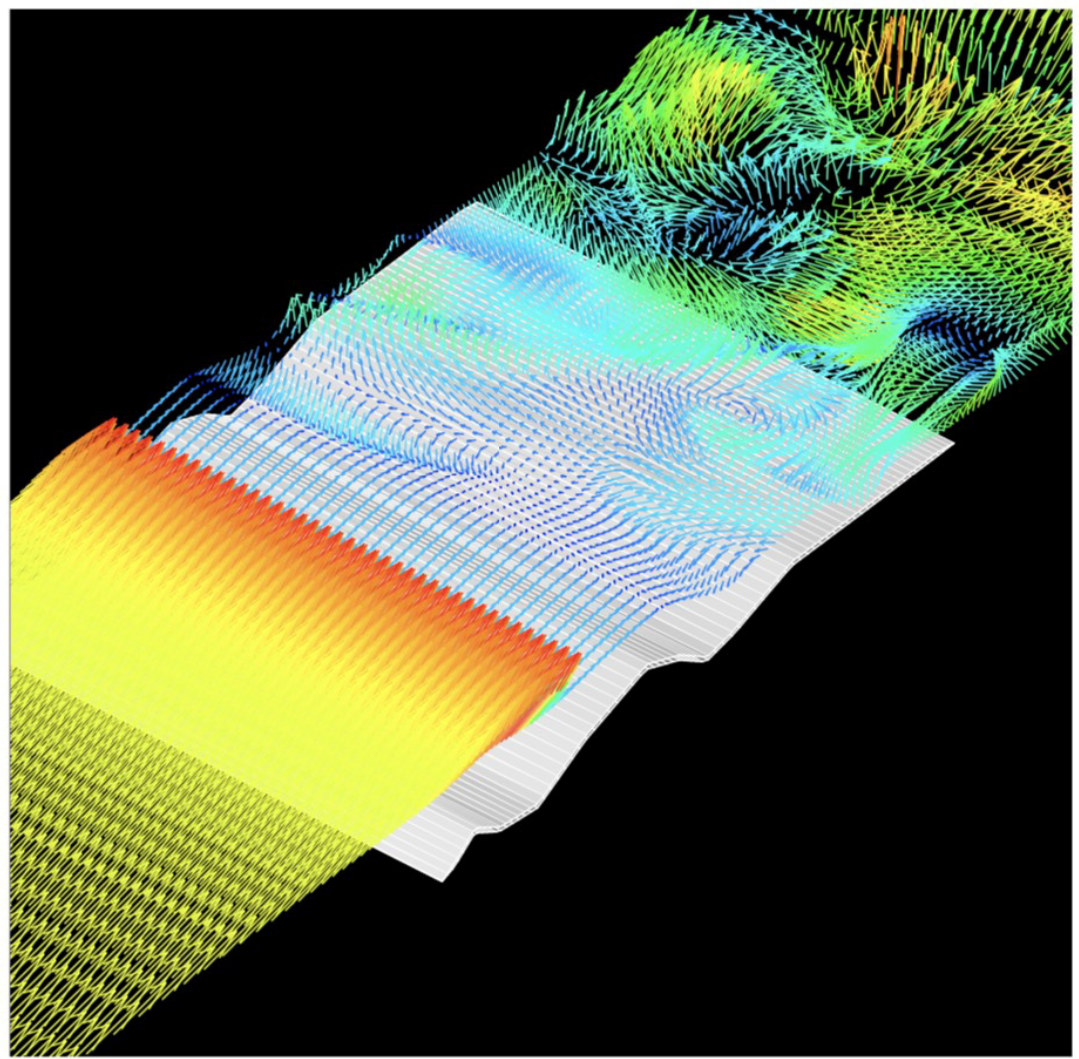

(a)

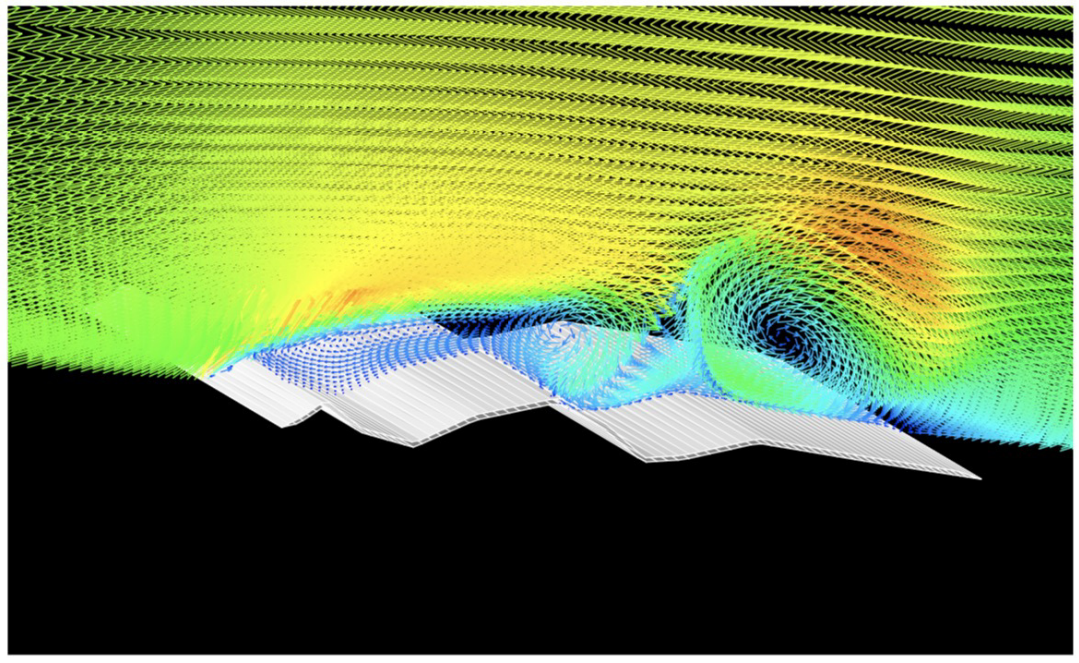

(b)

Figure 15. Instantaneous velocity field on the upper surface of the corrugated airfoil at angle of attack of $8^{\circ}, R e=4000$ : (a) top view; and (b) front view.

Figure 16 shows the values of the lift and drag coefficient of the two-dimensional and three-dimensional calculations for the corrugated airfoil and the profiled NACA2408 airfoil at Reynolds number $R e=4000$ when the angle of attack is varied from $0^{\circ}$ to $12^{\circ}$. Based on the above discussion, the values of the lift and drag coefficient are fluctuating with time at high angles of attack, but here the averaged values are shown in the present figures. It can be seen that the $2 \mathrm{D}$ calculation and $3 \mathrm{D}$ calculation, the corrugated airfoil and profiled NACA2408 airfoil all have a distinct lift and drag. First, comparing the results of 2D and 3D calculations, the difference is clearly shown: the lift and drag coefficient values for both the corrugated and the profiled NACA2408 airfoils are smaller in the 
3D calculation than the values in the 2D calculation at all angles of attack. This is due to the strong influence of the three-dimensionality of the flow field, as described in Figure 15. Next, when comparing the three-dimensional calculation of the corrugated airfoil with the three-dimensional calculation of the NACA2408 airfoil, the corrugated airfoil shows larger value in both the lift and drag coefficient at all angles of attack and has different characteristics at low angles of attack.

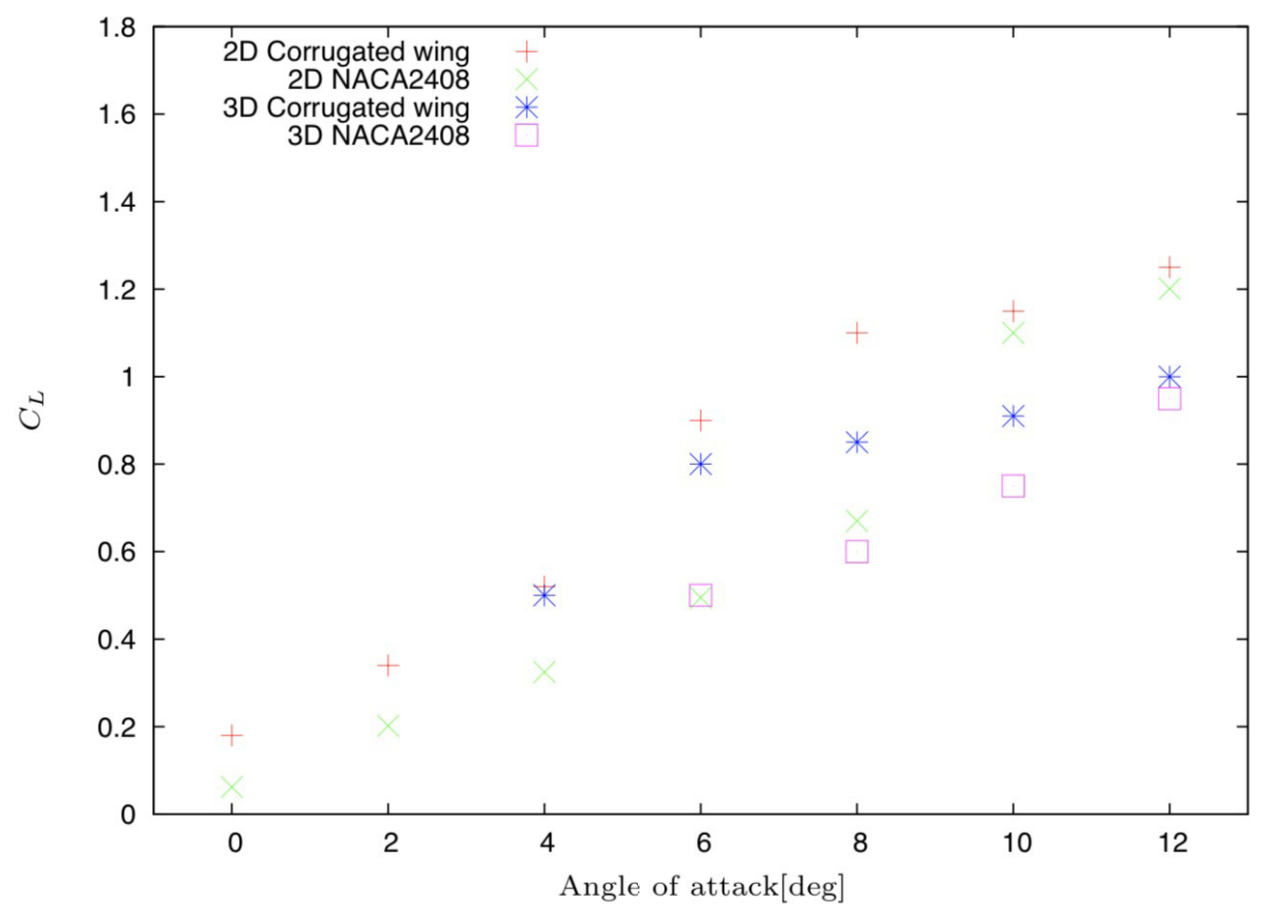

(a)

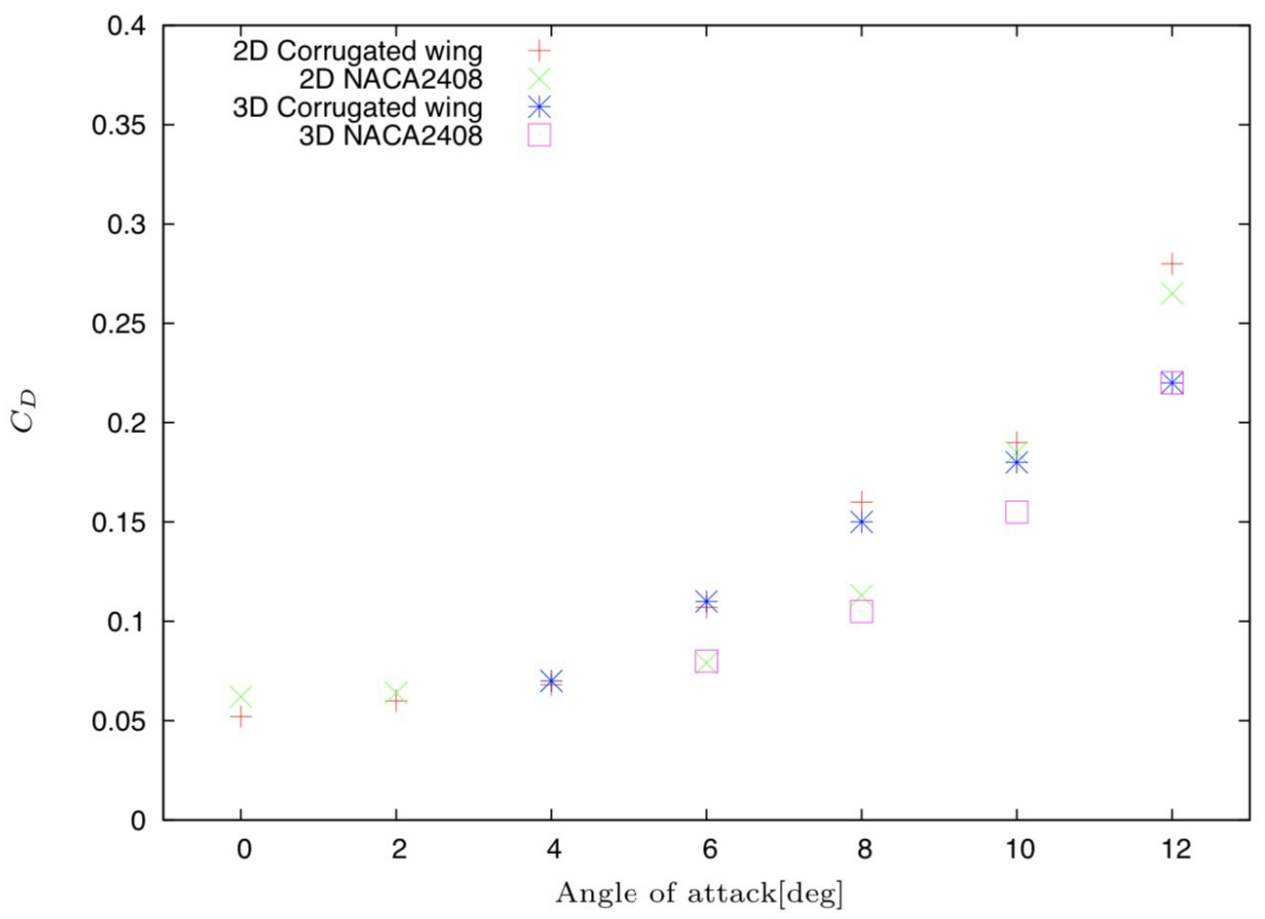

(b)

Figure 16. The averaged lift and drag coefficient of 2D and 3D calculations for the corrugated airfoil and NACA2408 airfoil at $R e=4000$ with varying angle of attack: (a) lift coefficient; and (b) drag coefficient. 
Figure 17 shows the mean lift-to-drag ratio of the 3D calculations for the corrugated airfoil and the profiled NACA2408 airfoil at $R e=4000$ with varying angle of attack. From this, it can be seen that, when the angle of attack is low, from $0^{\circ}$ to $6^{\circ}$, the lift-to-drag ratio of the corrugated airfoil is much larger than that of NACA2408 airfoil, and is equal at angles of attack of $8^{\circ}$ or more. As a result, the corrugated dragonfly airfoil is an effective wing shape for glide at a low angle of attack. However, the corrugated airfoils are also characterized by the fact that the lift and drag tend to fluctuate when the angle of attack is raised.



Figure 17. The mean lift-to-drag ratio of the 3D calculation for the corrugated airfoil and the 3D profiled NACA2408 airfoil at $R e=4000$ with varying angle of attack.

5.3. Aerodynamic Characteristics of Three-Dimensional Corrugated Airfoil under ThreeDimensional Calculation

In this section, we show the results of three-dimensional calculation of the flowing fluid around a three-dimensional corrugated wing with unevenness in the spanwise direction. The calculation area and calculation conditions are the same as those described for the 3D calculation of the 2D airfoil, and the target is a three-dimensional corrugated wing, as shown in Figure 18. The length in the spanwise direction is $0.6 \mathrm{~L}$, where $\mathrm{L}$ is the chord length, and an uneven pattern with two cycles is provided in the spanwise direction. This was determined by estimating the size of the 3D vortex from the visualization of the flow field around the 2D airfoil in the 3D calculation, as shown in Figure 15. The calculation was performed at an angle of attack of $2^{\circ}$ as a representative of low angles of attack and $8^{\circ}$ as a representative of high angles of attack, and the 3D calculation results of a 3D corrugated airfoil were compared with the 3D calculation results of a 2D corrugated airfoil. 


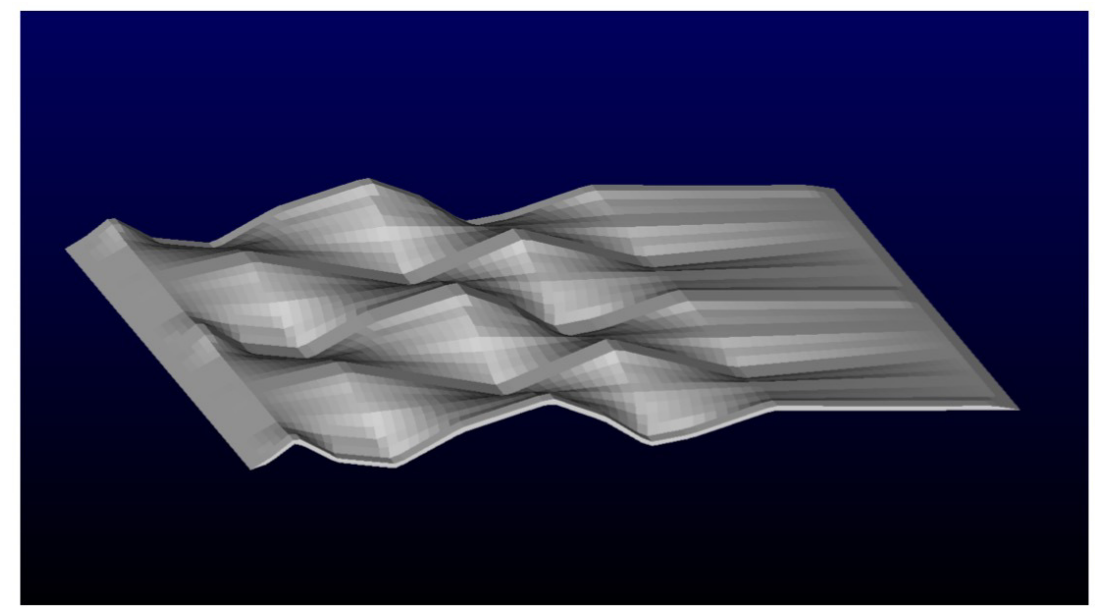

Figure 18. A three-dimensional corrugated wing shape.

Figure 19 shows the instantaneous velocity field on the upper surface of the three-dimensional corrugated wing at angle of attack of $2^{\circ}$ and $R e=4000$. It can be seen that the flow field around the $3 \mathrm{D}$ corrugated airfoil is almost steady, and the vortex stagnates at the dent of the wing, similar to the case of the 2D wing. Figure 20 shows the time history of lift coefficient of $3 \mathrm{D}$ calculations for the 2D and 3D corrugated airfoil at $R e=2000$ and 4000 with angle of attack of $8^{\circ}$. Here, the lift coefficient is averaged over the entire corrugated wing. From the results, there was little difference between the average value and the amount of temporal fluctuation of the lift coefficient between the 2D and 3D corrugated airfoil in the 3D calculation. For instance, in the case of $R e=2000$, as shown in Figure 20a, when the flow field is sufficiently developed (after the dimensionless time 40), the mean value of $C_{L}$ for $2 \mathrm{D}$ and $3 \mathrm{D}$ corrugated airfoils is almost the same, and the fluctuations of $C_{L}$ of $3 \mathrm{D}$ corrugated airfoil is smaller compared with that of 2D corrugated airfoil. In the case of $R e=4000$, as shown in Figure 20b, the $3 \mathrm{D}$ corrugated airfoil shows the same smaller fluctuations of $C_{L}$. As a result, it was confirmed that the unevenness in the spanwise direction for a three-dimensional corrugated wing promotes the three-dimensional flow field around the three-dimensional wing and suppresses the lift fluctuations of the entire corrugated wing.

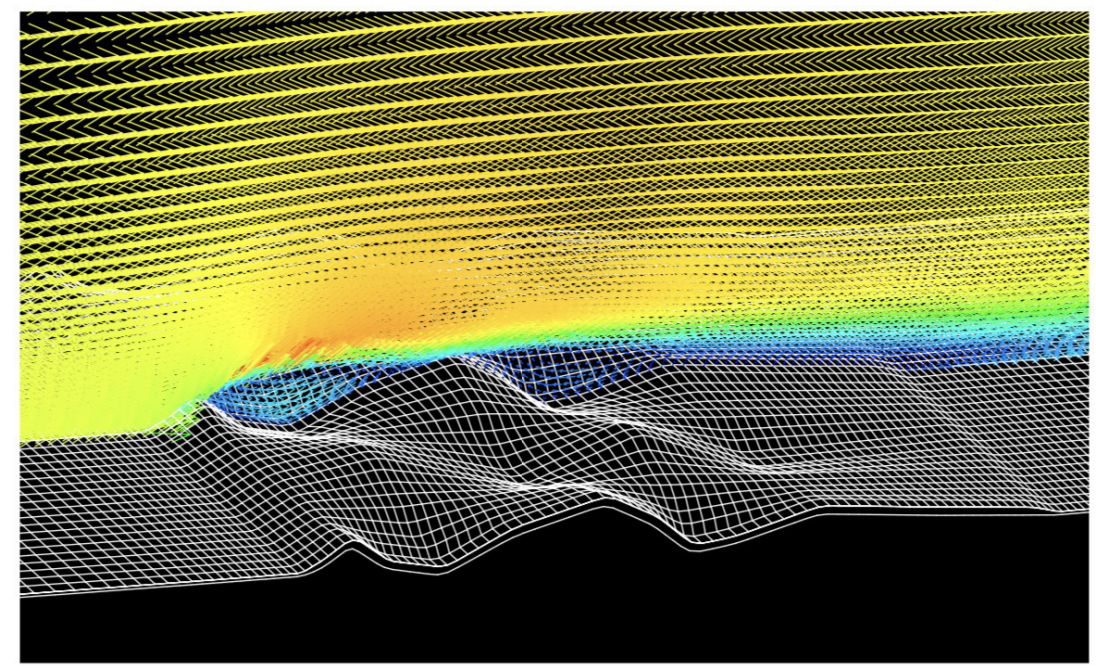

Figure 19. Instantaneous velocity field on the upper surface of the three dimensional corrugated wing at angle of attack of $2^{\circ}$ and $\operatorname{Re}=4000$. 


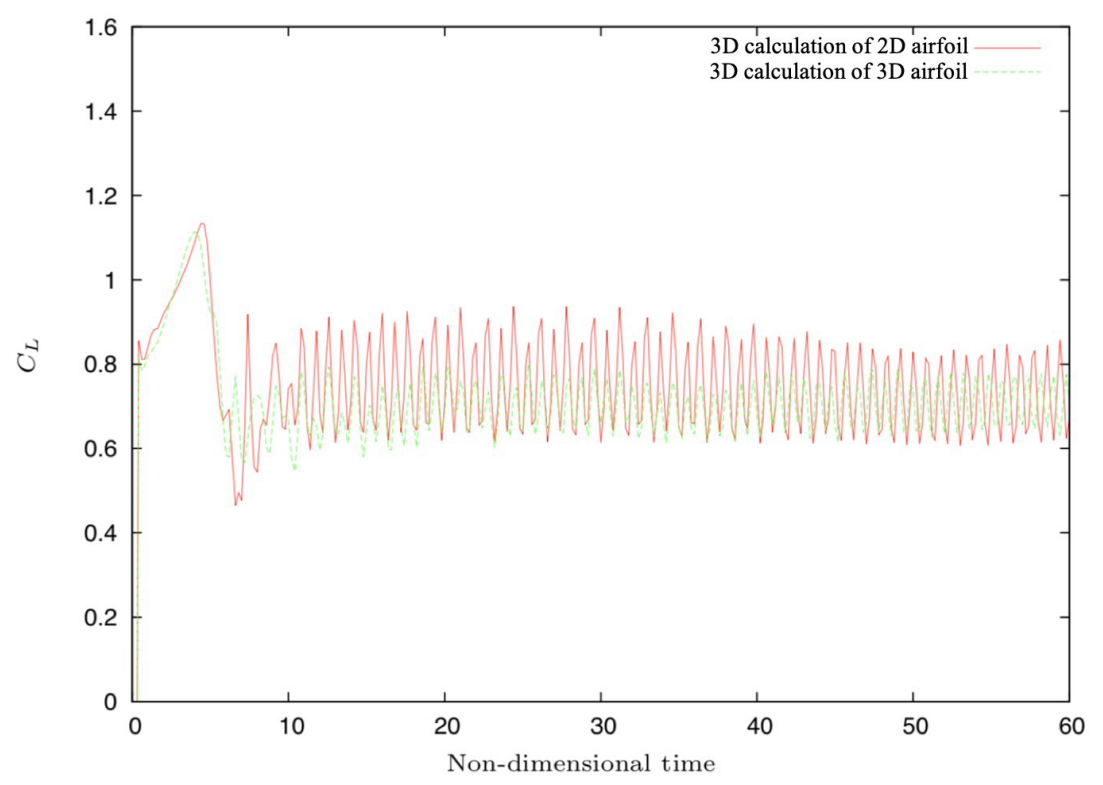

(a)

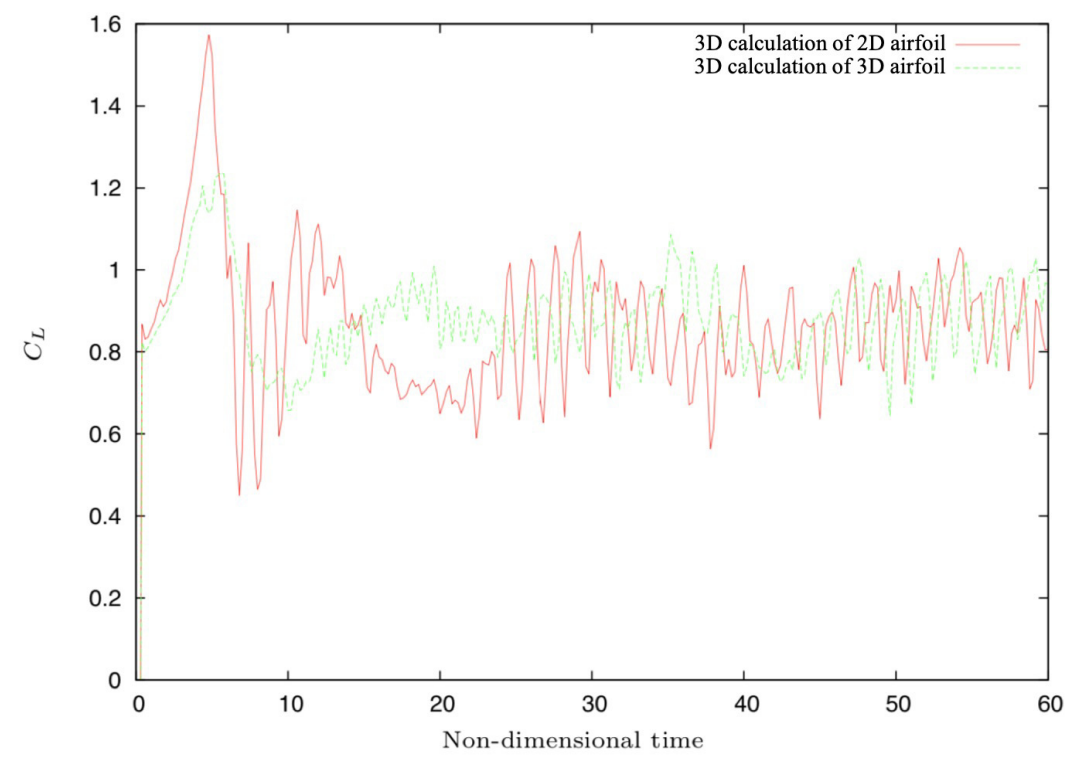

(b)

Figure 20. Time history of lift coefficient of $3 \mathrm{D}$ calculations for the $2 \mathrm{D}$ and $3 \mathrm{D}$ corrugated airfoil at $R e=2000$ and 4000 with angle of attack of $8^{\circ}:$ (a) $R e=2000$; and (b) $R e=4000$.

\subsection{Hydrodynamic Moment and Attitude Stability}

\subsubsection{Fixed Angle of Attack}

In a steady-state with the airfoil fixed at an angle of attack in a uniform flow, the moment coefficient CM due to the fluid force generated around the position of the airfoil chord length of $25 \%$ from the airfoil leading edge was evaluated. The static moment coefficients were averaged over time after the flow field was fully developed. Figure 21 shows the mean static moment coefficient due to the fluid force at each angle of attack for the corrugated airfoil and the profiled NACA2408 airfoil at $R e=4000$. Here, the angle of attack is varied by $1^{\circ}$ from $-6^{\circ}$ to $6^{\circ}$. The hydrodynamic moment acting on the airfoil increases as the angle of attack increases, and the hydrodynamic moment increases in the direction of decreasing the angle of attack, and then it is called that the airfoil in the range of angle of attacks is stable. In Figure 21, the region where the angle of attack $\alpha$ is statically stable is approximately 
$-4^{\circ}<\alpha<4^{\circ}$ for the profiled NACA2408 airfoil, while $-2^{\circ}<\alpha<2^{\circ}$ for the corrugated airfoil. In addition, through the least square approximation, the rate of change in hydrodynamic coefficient over angle of attacks in Figure 21 is 0.064 and 0.015 for the profiled NACA2408 airfoil in range of $-4^{\circ}<\alpha<4^{\circ}$ and the corrugated airfoil in range of $-2^{\circ}<\alpha<2^{\circ}$, respectively. As a result, the profiled NACA2408 airfoil is statically more stable near the angle of attack of $0^{\circ}$ and has a wider static stable range of the angle of attack compared with the corrugated airfoil. Meanwhile, for variations of angle of attack in the static stable region, a lager hydrodynamic moment fluctuation is obtained in the direction to make it smaller for the profiled NACA2408 airfoil.



Figure 21. The mean static moment coefficient of the corrugated airfoil and the profiled NACA2408 airfoil at $R e=4000$ with varying angle of attack.

\subsubsection{Angle of Attack Passively Changed by the Fluid Force}

A constant supporting moment $M_{0}$ is applied from the leading edge of the airfoil around the rotating support point with a chord length of $25 \%$ so that it balances the hydrodynamic moment due to the fluid force generated at the initial angle of attack. Here, the initial angle of attack is $0^{\circ}$, thus, based on the results in Figure 21, $C_{M 0}$ for the NACA2408 airfoil and the corrugated airfoil are 0 and -0.0393 , respectively. After applying an initial disturbance with a small angle $\Delta \alpha=+0.001^{\circ}$ for the objective airfoils, the time evolution of the angle of attack of the objective airfoil is observed. The moment of inertia for the airfoil is used to evaluate the influence of flow fields around differently shaped airfoils on the dynamic stability of the angle of attack. The purpose of this study was to evaluate the influence of the difference of the airfoil shape on the fluctuation of the angle of attack by comparing the time evolution of the angle of attack after a small angle disturbance. To remove the effect of the moment of inertia, the density ratio between the structure and the fluid is set so that the moment of inertia of the corrugated airfoil and the NACA2408 airfoil is equal. For the density ratio between the corrugated airfoil and the fluid $r_{\rho C}=3.0 \times 10^{-3}$, Figure 22 shows the time evolution of angle of attack and pitching moment coefficient of fluid force for the corrugated airfoil and NACA2408 airfoil at $R e=4000$ with the initial angle of attack of $0^{\circ}$. As shown in the solid line in Figure 22, the angle of attack of both kinds of airfoils oscillates with increasing amplitude of around $0^{\circ}$ over time. The angle of attack of the corrugated airfoil exceeded the range of static stability (see Figure 21, $-2^{\circ}<\alpha<2^{\circ}$ ) after dimensionless time 390 and diverged in the positive direction. Therefore, we compared the fluctuations of the angle of attack of the corrugated airfoil and NACA2408 airfoil up to dimensionless time 390. To further understand the relationship between the difference in wing shape and the fluctuations in the attack angle, the distribution of the relative moment coefficient between the moment by the fluid 
force and the support moment $M_{0}$ is also shown by a broken line in Figure 22. From the results in the figure, it can be confirmed that the corrugated airfoil has a larger amplitude of fluctuations of the angle of attack and larger amplitude of the relative moment coefficient compared with the profiled NACA2408 airfoil. In addition, there is a dimensionless time delay of the moment of fluid force with respect to fluctuations in the angle of attack, especially when the distribution of the moment coefficient and the angle of attack takes an extreme value or $0^{\circ}$.

The mean value of the dimensionless time delay of extreme values and $0^{\circ}$ for the moment coefficient with respect to fluctuations in the angle of attack and the coefficient of variation obtained by dividing the standard deviation of the dimensionless time delay by the mean value of the dimensionless time delay are summarized in Table 4. It can be seen that the dimensionless time delay of the corrugated airfoil is significantly greater than that of the NACA2408 airfoil. Although there are slight fluctuations, the dimensionless time delay for both two airfoils is concentrated near its mean value. Regarding the moment coefficient when the angle of attack is $0^{\circ}$, the moment coefficient is negative due to the dimensionless time delay, as the angle of attack changes from negative to positive. Thus, the airfoil has a hydrodynamic moment in the direction of increasing the angle of attack. Similarly, when the angle of attack changes from positive to negative, the moment coefficient is positive due to the dimensionless time delay, and then a hydrodynamic moment is generated in the direction of increasing the fluctuations of the angle of attack. As a result, based on the above analysis, the increase in the amplitude of fluctuations of the angle of attack is more pronounced for the corrugated airfoil, which has larger dimensionless time delay than the NACA2408 airfoil. Actually, the solid line in Figure 22 verifies the results of the analyses.

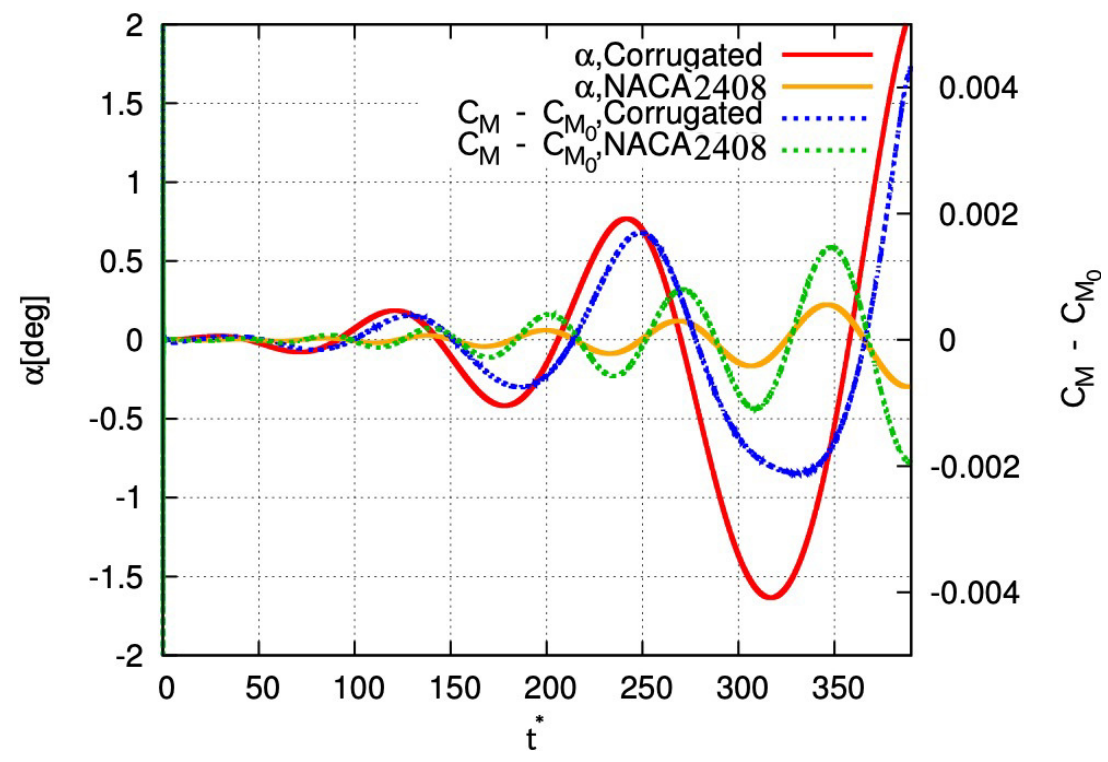

Figure 22. Time evolution of angle of attack and pitching relative moment coefficient of fluid force for the corrugated airfoil and NACA2408 airfoil at $R e=4000$, with initial angle of attack of $0^{\circ}$ $\left(r_{\rho C}=3.0 \times 10^{-3}\right)$.

Table 4. Dimensionless time delay between angle of attack and moment force.

\begin{tabular}{ccc}
\hline Dimensionless Time Delay & Corrugated & NACA2408 \\
\hline Average & 7.8 & 2.3 \\
Coefficient of Variation & 0.038 & 0.13 \\
\hline
\end{tabular}

From the numerical calculation results of the problem setting with a fixed angle of attack, it was predicted that the attitude of the airfoil would be stabilized even if a small disturbance of the angle of attack was applied. However, when the angle of attack was passively changed by the fluid force for 
the airfoils around the rotating support point with a chord length of $25 \%$ from the leading edge, the numerical results were different from this expectation. Therefore, we discuss the destabilization of the attack angle of the airfoil by modeling the motion of a rotationally supported airfoil that receives fluid force. In the distribution of the hydrodynamic moment coefficient for the fixed angle of attacks (see Figure 21), the distribution is linear near the angle of attack of $0^{\circ}$ for both two airfoils. Thus, in Equation (3), $C_{M}-C_{M 0}$ can be linearized with respect to the angle of attack $\alpha$ by performing Taylor expansion (up to the first order) with the angle of attack near $0^{\circ}$. Thus,

$$
-I \frac{d^{2} \theta}{d t^{2}} \simeq \frac{1}{2} u_{u n i}^{2} S\left(C_{M}-\left.C_{M_{0}}\right|_{\alpha=0}+\left.\frac{\partial C_{M}}{\partial \alpha}\right|_{\alpha=0} \alpha\right) .
$$

Here, based on the above problem setting, the support hydrodynamic moment coefficient $C_{M_{0}}$ is given so that $C_{M}-C_{M_{0}}=0$ at the angle of attack $\alpha=0^{\circ}$, thus $C_{M}-\left.C_{M_{0}}\right|_{\alpha=0}=0$. As shown in Figure 23, for the airfoil rotating unsteadily, the angle of airfoil attitude $\theta$ is distinguished from the actual angle of attack $\alpha . l$ is the length that represents the velocity derived from the rotational motion, and $l=0.25 \mathrm{~L}$. The geometric relationship between velocity and angle of attitude $\theta$ and attack $\alpha$ is shown in Figure 24. $u_{r e l}$ represents the relative velocity between the uniform inflow velocity $u_{u n i}$ and the velocity of length $l$ due to the rotation, see Figure 24 . When $\theta$ is sufficiently small, according to the geometrical relationship in Figure 24,

$$
\tan (\theta-\alpha) \simeq \frac{i \dot{\theta}}{u_{u n i}} \Rightarrow \alpha \simeq \theta-\frac{l}{u_{u n i}} \dot{\theta}
$$

Applying Equation (8) to Equation (7) leads to

$$
-I \frac{d^{2} \theta}{d t^{2}} \simeq \frac{1}{2} u_{u n i}^{2} S\left[\left.\frac{\partial C_{M}}{\partial \alpha}\right|_{\alpha=0}\left(\theta-\frac{l}{u_{u n i}} \dot{\theta}\right)\right] .
$$

Dividing Equation (9) by $1 / 2 u_{u n i}^{2} S$ and using $\hat{I}=I / 2 u_{u n i}^{2} S$ and $\left.\frac{\partial C_{M}}{\partial \alpha}\right|_{\alpha=0}=\beta$ results in

$$
-\hat{I} \ddot{\theta}+\beta \frac{l}{u_{u n i}} \dot{\theta}+\beta \theta=0 .
$$

Performing Laplace transform on both sides of Equation (10) and using $\Theta(s)=\mathcal{L}(s)$ lead to

$$
\hat{I}\left(s^{2} \Theta(s)-s \theta(0)-\dot{\theta}(0)\right)-\beta \frac{l}{u_{u n i}}(s \Theta(s)-\theta(0))+\beta \Theta(s)=0 .
$$

According to the above problems setting of the initial disturbance of the angle of attack at $t=0$, $\theta(0)=1.0 \times 10^{-3}$ and $\dot{\theta}(0)=0$ can be obtained. Then, rearrangement of Equation (11) yields

$$
\frac{\Theta(s)}{\theta(0)}=\frac{\left(s-\beta \frac{l}{u_{u n i}}\right)}{\hat{I} s^{2}-\beta \frac{l}{u_{u n i}} s+\beta} .
$$

From Equation (12), the characteristic polynomial of this control system (a rotationally supported airfoil that receives fluid force) can be obtained from the fraction part on the right-hand side of Equation (12), i.e., $P(s)=\hat{I} s^{2}-\beta \frac{l}{u_{u n i}} s+\beta$. Since the characteristic polynomial of this control system is a second-order polynomial, the stability can be easily evaluated using Routh-Hurwitz criterion [33,34]. The necessary and sufficient condition of the Routh-Hurwitz criterion for a second-order polynomial is that all coefficients of the polynomial must be greater than zero. For the constant in the polynomial $P(s)=\hat{I} s^{2}-\beta \frac{l}{u_{u n i}} s+\beta, \hat{I}$ is an estimate of the dimensionless moment of inertia around the rotational support point of the airfoil, the time scale expressed by $\frac{l}{u_{u n i}}$ is 10 , and $\beta$ is the slope of the interval where the distribution of the fluid force moment coefficient is linear in 
Figure 21, that is, it is 0.015 and 0.064 for the corrugated airfoil and the NACA2408 airfoil, respectively. In conclusion, $\hat{I}>0,-\beta \frac{l}{u_{u n i}}<0$ and $\beta>0$. According to the Routh-Hurwitz stability criterion for a second-order polynomial, the control system of the angle of attack passively changed by the fluid force for both two airfoils (around the rotating support point) can be said to be an unstable system.

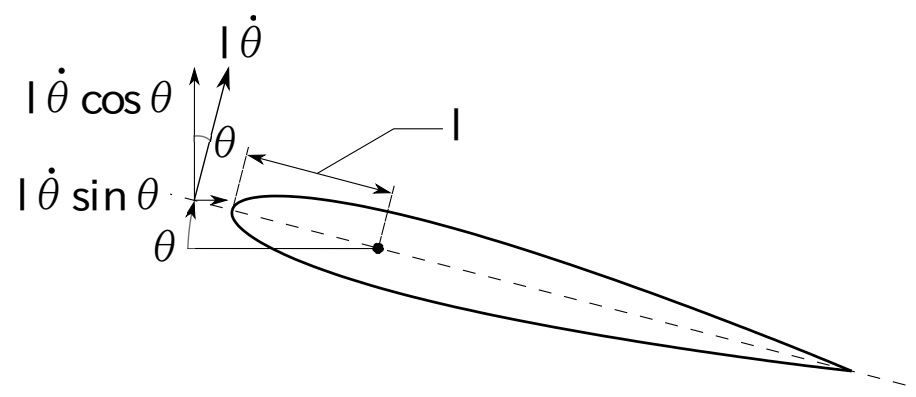

Figure 23. Attitude and rotation speed of an airfoil.

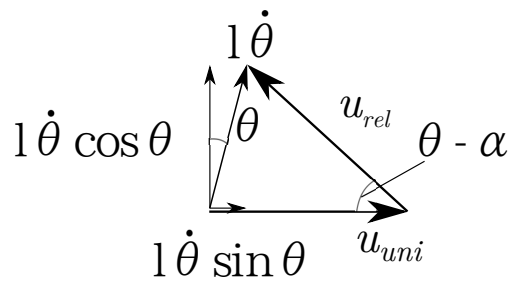

Figure 24. Geometric relationship between velocity and angle of attitude and attack.

\section{Conclusions}

In this study, two-dimensional and three-dimensional numerical simulations were conducted to investigate the aerodynamic characteristics, especially the unsteady aerodynamic characteristics and attitude stability, of a bio-inspired corrugated airfoil compared with a smooth-surfaced airfoil (NACA2408 airfoil) at the chord Reynolds number of 4000 to explore the potential applications of non-traditional, corrugated dragonfly airfoils for small MAVs or UAVs designs. There were two problem settings applied to our numerical calculations. First, the airfoil was fixed at a constant angle of attack to analyze the aerodynamic characteristics and the hydrodynamic moment of the corrugated airfoil and smooth-surfaced airfoil. Especially, the unsteady behavior of lift and drag coefficient was studied. Second, to consider the influence of the fluid force acting on the airfoil surface, the angle of attack of airfoils was passively changed by the fluid force to analyze the attitude stability. The current numerical solver for the flow field around an unsteady rotating airfoil when the angle of attack is passively changed by the fluid force was validated in comparison with the numerical data from Kaneko et al. [30]. Satisfactory results were obtained. It was confirmed that the corrugated airfoil performs (in terms of the lift-to-drag ratio) much better than the profiled NACA2408 airfoil at low Reynolds number $R e=4000$ in low angle of attack range of $0^{\circ}-6^{\circ}$, and performs as well at the angle of attack of $6^{\circ}$ or more. At these low angles of attack, although the pleated airfoil experiences an increase in the pressure drag, it is more than compensated by a concomitant decrease in the friction shear drag. The reduction in the shear drag is due to the fact that there exist recirculation zones inside the cavities formed by the pleats, and they lead to negative shear drag contribution. Furthermore, the corrugated airfoil could provide larger lift than the profiled NACA2408 airfoil at a low angle of attack. The increase in the lift is due to the fact that the negative pressure produced at the valleys of the corrugated airfoil contributes to the increased lift. It was found that, when the angle of attack is high, the lift and drag in the two-dimensional numerical calculation for the corrugated airfoil and the profiled NACA2408 airfoil fluctuate strongly, especially for the corrugated airfoil. However, in three-dimensional simulation, especially for a three-dimensional corrugated airfoil with unevenness in the spanwise direction, smaller fluctuations and the smaller average value in the lift and drag were obtained than the results in 2D 
calculations. The reason was found that the flow becomes three-dimensional in the separated shear layer when the angle of attack is high. In particular, a three-dimensional wing with irregularities in the spanwise direction can promote three-dimensional flow and can suppress lift fluctuations even at high angles of attack. On the other hand, the attitude stability of the corrugated airfoil was studied. Regarding the airfoil fixed at angles of attack, the profiled NACA2408 airfoil is statically more stable near the angle of attack of $0^{\circ}$ and has wider static stable range of the angle of attack compared with the corrugated airfoil, such as $-4^{\circ}<\alpha<4^{\circ}$ for the profiled airfoil and $-2^{\circ}<\alpha<2^{\circ}$ for the corrugated airfoil. Regarding the airfoil with the angle of attack passively changed by the fluid force, the corrugated airfoil has a larger amplitude of fluctuations of angle of attack, a larger amplitude of the relative moment coefficient, and a larger dimensionless time delay of the moment of fluid force with respect to fluctuations in the angle of attack compared with the profiled NACA2408 airfoil. Finally, based on the Routh-Hurwitz stability criterion, it was confirmed that the systems of the angle of attack passively changed by the fluid force for both two airfoils (around the rotating support point) are unstable systems.

Author Contributions: H.T. and Y.L. conceived the original ideal; H.T. wrote and edited the manuscript; and Y.L., X.L. and Y.F. supervised the study.

Funding: This research was funded by the project of Chinese National Natural Science Foundation (grant number: 51575220), the project of the Key Scientific and Technological Project of Jilin Province (grant numbers: 20160519008JH and 20170204073GX), and the project of National Key R\&D Program of China (grant number: 2016YFB0101402).

Acknowledgments: The authors thank Takeo Kajishima and Kei Okabayashi for their help with the numerical method proposed and simulations conducted.

Conflicts of Interest: The authors declare no conflict of interest.

\section{Abbreviations}

The following abbreviations are used in this manuscript:

2D two-dimensional

3D three-dimensional

MAVs micro air vehicles

SOR successive over-relaxation

UAVs unmanned aerial vehicles

\section{References}

1. Mohiuddin, A.; Taha, T.; Zweiri, Y.; Gan, D. UAV payload transportation via RTDP based optimized velocity profiles. Energies 2019, 12, 3049. [CrossRef]

2. Estevez, J.; Lopez-Guede, J.M.; Grana, M. Quasi-stationary state transportation of a hose with quadrotors. Robot. Auton. Syst. 2015, 63, 187-194. [CrossRef]

3. Wall Street Journal. Google Drones Can Already Deliver You Coffee In Australia. Available online: https: / / www.youtube.com/watch?v=prhDrfUgpB0 (accessed on 6 April 2019)

4. Stolaroff, J.K.; Samaras, C.; O’Neill, E.R.; Lubers, A.; Mitchell, A.S.; Ceperley, D. Energy use and life cycle greenhouse gas emissions of drones for commercial package delivery. Nat. Commun. 2018, 9, 409. [CrossRef] [PubMed]

5. Carmichael, B.H. Low Reynolds Number Airfoil Survey, Volume 1; NASA CR-165803; NASA: Washington, DC, USA, 1981

6. Lissaman, P.B.S. Low-Reynolds-number airfoils. Annu. Rev. Fluid Mech. 1983, 15, 223-239. [CrossRef]

7. Gad-el-Hak, M. Micro-air-vehicles: Can they be controlled better? J. Aircr. 2001, 38, 419-429. [CrossRef]

8. Wakeling, J.M.; Ellington, C.P. Dragonfly flight. I. Gliding flight and steady-state aerodynamic forces. J. Exp. Biol. 1997, 200, 543-556. [PubMed]

9. Kesel, A.B. Aerodynamic characteristics of dragonfly wing sections compared with technical aerofoils. J. Exp. Biol. 2000, 203, 3125-3135. 
10. Rees, C.J. Form and function in corrugated insect wings. Nature 1975, 256, 200-203. [CrossRef]

11. Brodsky, A.K. The Evolution of Insect Flight; Oxford University Press: Oxford, UK, 1994.

12. Rudolph, R. Aerodynamic properties of Libellula quadrimaculata L.(Anisoptera: Libellulidae), and the flow around smooth and corrugated wing section models during gliding flight. Odonatologica 1978, 7, 49-58.

13. Okamoto, M.; Yasuda, K.; Azuma, A. Aerodynamic characteristics of the wings and body of a dragonfly. J. Exp. Biol. 1996, 199, 281-294.

14. Luo, G.; Sun, M. The effects of corrugation and wing planform on the aerodynamic force production of sweeping model insect wings. Acta Mech. Sin. 2005, 21, 531-541. [CrossRef]

15. Vargas, A.; Mittal, R.; Dong, H. A computational study of the aerodynamic performance of a dragonfly wing section in gliding flight. Bioinspir. Biomim. 2008, 3, 026004. [CrossRef] [PubMed]

16. Murphy, J.T.; Hu, H. An experimental study of a bio-inspired corrugated airfoil for micro air vehicle applications. Exp. Fluids 2010, 49, 531-546. [CrossRef]

17. Khan, M.D.; Padhy, C.; Nandish, M.; Rita, K. Computational Analysis of Bio-Inspired Corrugated Airfoil with Varying Corrugation Angle (16 February 2018). Available online: https:/ / ssrn.com/abstract=3124809 (accessed on 22 October 2019).

18. Uppu, S.P.; Manisha, D.; Devi, G.D.; Chengalwa, P.; Devi, B.V. Aerodynamic Analysis of a Dragonfly. J. Adv. Res. Fluid Mech. Therm. Sci. J. 2018, 1, 31-41.

19. Mohamed, A.; Massey, K.; Watkins, S.; Clothier, R. The attitude control of fixed-wing MAVS in turbulent environments. Prog. Aerosp. Sci. 2014, 66, 37-48. [CrossRef]

20. Orr, M.W.; Rasmussen, S.J.; Karni, E.D.; Blake, W.B. Framework for developing and evaluating MAV control algorithms in a realistic urban setting. In Proceedings of the IEEE 2005 American Control Conference, Portland, OR, USA, 8-10 June 2005; pp. 4096-4101.

21. Galinski, C.E.Z.A.R.Y.; Zbikowski, R. Some problems of micro air vehicles development. Bull. Pol. Acad. Sci. Tech. Sci. 2007, 55, 91-98.

22. Mohamed, A.; Clothier, R.; Watkins, S.; Sabatini, R.; Abdulrahim, M. Fixed-wing MAV attitude stability in atmospheric turbulence, part 1: Suitability of conventional sensors. Prog. Aerosp. Sci. 2014, 70, 69-82. [CrossRef]

23. Chorin, A.J. A numerical method for solving incompressible viscous flow problems. J. Comput. Phys. 1967, 2, 12-26. [CrossRef]

24. Tamura, A.; Kikuchi, K.; Takahashi, T. Residual cutting method for elliptic boundary value problems. J. Comput. Phys. 1997, 137, 247-264. [CrossRef]

25. Kajishima, T.; Ohta, T.; Okazaki, K.; Miyake, Y. High-order finite-difference method for incompressible flows using collocated grid system. JSME Int. J. Ser. B Fluids Therm. Eng. 1998, 41, 830-839. [CrossRef]

26. Ohta, T.; Kajishima, T. Analysis of non-steady separated turbulent flow in an asymmetric plane diffuser by direct numerical simulations. J. Fluid Sci. Technol. 2010, 5, 515-527. [CrossRef]

27. Tang, H.; Lei, Y.; Fu, Y. Noise Reduction Mechanisms of an Airfoil with Trailing Edge Serrations at Low Mach Number. Appl. Sci. 2019, 9, 3784. [CrossRef]

28. Obata, A.; Sinohara, S. Flow visualization study of the aerodynamics of modeled dragonfly wings. AIAA J. 2009, 47, 3043-3046. [CrossRef]

29. Thompson, J.F.; Soni, B.K.; Weatherill, N.P. Handbook of Grid Generation; CRC Press: Boca Raton, FL, USA, 1998.

30. Kaneko, Y.; Omori, K.; Kajishima, T. Numerical study on the aerodynamics of an airfoil moving close to an air-water interface. Trans. Jpn. Soc. Mech. Eng. 2016, 82, 16-00112.

31. Anderson, J.M.; Streitlien, K.; Barrett, D.S.; Triantafyllou, M.S. Oscillating foils of high propulsive efficiency. J. Fluid Mech. 1998, 360, 41-72. [CrossRef]

32. Nakae, Y.; Ohtake, T.; Muramatsu, A.; Motohashi, T. Three dimensionalization of the Flow Field around a NACA0012 Airfoil at Low Reynolds Numbers. J. Jpn. Soc. Aeronaut. Space Sci. 2011, 59, 244-251. 
33. Routh, E.J. A Treatise on the Stability of a Given State of Motion: Particularly Steady Motion; Macmillan and Company: Troon, Scotland, 1877.

34. Hurwitz, A. Ueber die Bedingungen, unter welchen eine Gleichung nur Wurzeln mit negativen reellen Theilen besitzt. Mathem. Ann. 1895, 46, 273-284. [CrossRef] 